\title{
1 Cassette recruitment in the chromosomal Integron of Vibrio \\ 2 cholerae
}

3 Claire Vit ${ }^{1,2,3,6}$, Egill Richard ${ }^{1,2,3}$, Florian Fournes $^{1,2}$, Clémence Whiteway ${ }^{1,2,7}$, Xavier 4 Eyer ${ }^{1,2,8}$, Delphine Lapaillerie ${ }^{4,5}$, Vincent Parissi ${ }^{4,5}$, Didier Mazel ${ }^{1,2, *}$ and Céline Loot ${ }^{1,2, *}$

\footnotetext{
${ }^{1}$ Institut Pasteur, Unité de Plasticité du Génome Bactérien, Département Génomes et Génétique, 28 Rue du Dr Roux, 75015 Paris, France.

${ }^{2}$ CNRS, UMR3525, 28 Rue du Dr Roux, 75015 Paris, France.

${ }^{3}$ Sorbonne Université, Collège doctoral, F-75005 Paris, France.

${ }^{4}$ University of Bordeaux, Fundamental Microbiology and Pathogenicity Laboratory, CNRS, UMR 5234, SFR TransBioMed, Bordeaux, France.

${ }^{5}$ Viral DNA Integration and Chromatin Dynamics Network (DyNAVir), France.

${ }^{6}$ Institut de Cancérologie Strasbourg Europe ICANS, 3 Rue de la porte de l'hôpital, 67065 Strasbourg, France.

${ }^{7}$ Vrije Universiteit Brussel and Flanders Institute for Biotechnology, Microbial Resistance and Drug Discovery laboratory, Center for Structural Biology, Pleinlaan 2, Building E-3, 1050 Brussels, Belgium.

${ }^{8}$ Hôpital Lariboisière, Assistance Publique des Hôpitaux de Paris, 2 rue Ambroise Paré, 75010 Paris, France.
}

\footnotetext{
* To whom correspondence should be addressed. Tel: +33 140613 287; Fax: +33 145688 834; Email: celine.loot@pasteur.fr. Correspondence may also be addressed to Didier Mazel; Tel: +33 14061 3284; Fax: +33 145688 834; Email: didier.mazel@pasteur.fr;
}

\section{ABSTRACT}

Integrons are genetic systems conferring to bacteria a rapid adaptation capability. The integron integrase is able to capture, stockpile and shuffle novel functions embedded in cassettes. This involves the recognition of both substrates, the attI site, and the cassette associated attC sites. Integrons can be sedentary and chromosomally located (SCI) or, carried by conjugative plasmids (Mobile Integron, MI), hence favoring their dissemination among bacteria. Here, for the first time, we investigate the cassette recruitment in the Vibrio cholerae SCI during conjugation and natural transformation. We demonstrated that horizontally transferred cassette can be recruited inside the chromosomal integron. The endogenous integrase expression is sufficiently triggered, after SOS response induction mediated by the entry of single-stranded cassettes during conjugation and natural transformation, to mediate significant cassette insertion. We demonstrate that the attIA insertion is preferential, despite the presence of 180 attC sites in the integron array. Thanks to the presence of a promoter in the attIA site vicinity, all these newly inserted cassettes are expressed and prone to adaptive 
selection. We also show that the RecA protein is critical for cassette recruitment in $V$. cholerae SCI but not in MIs. Moreover, a contrario to MIs, the $V$. cholerae SCI is not active in others bacterial hosts. MIs might have evolved from the SCIs by overcoming host factors, which would explain their large dissemination in bacteria and their role in the antibioresistance expansion.

\section{INTRODUCTION}

Mobile Genetic Elements (MGE) widely contribute to the evolution of bacterial genomes notably by conferring adaptive traits such as the ability to resist antibiotic treatments (Partridge et al., 2018). This can have dramatic consequences, especially when concerning pathogenic bacteria. Integrons are MGE that are considered as major contributors in the rise of multi-resistance in Gram-negative bacteria. These genetic systems were discovered in the late 80s and described as platforms involved in the capture, stockpiling and expression of antibiotic resistance genes, embedded in structures termed "cassettes" (Stokes and Hall, 1989). These latter were referred to as mobile integrons (MIs) because of their associations with transposable elements and conjugative plasmids. Larger integrons located on bacterial chromosomes were discovered later, the superintegron of $V$. cholerae being the first identified (Mazel et al., 1998). This superintegron is located on the chromosome 2 of $V$. cholerae and contains 180 gene cassettes coding mainly for proteins with no homologs in the databases or for proteins of unknown function. In contrast to their mobile counterpart and to refer at their location, such structures are termed Sedentary Chromosomal Integrons (SCI). They are common features of bacterial genomes from Vibrio genus and are generally distributed in several genomes of $\beta$ - and $\gamma$-proteobacteria (Cambray et al., 2010; Cury et al., 2016). These large SCIs were proposed to be the ancestors of MIs (Rowe-Magnus et al., 2001) and the stockpiling capacity from both types of integrons suggests that they may have distinct but complementary roles (Loot et al., 2017). Indeed, large SCIs such as those found in genomes of Vibrio species, could constitute a reservoir of gene cassettes that can be captured and spread by MIs (Loot et al., 2017; Rowe-Magnus et al., 2002).

Both types of integrons are composed of a stable platform and a variable cassette array. The platform contains a gene coding for a tyrosine-recombinase, IntI, the attI recombination site and the resident promoter $\mathrm{P}_{\mathrm{C}}$ oriented toward the variable cassette array (Figure $1 \mathrm{~A}$ ). The cassette array contains a pool of gene cassettes generally composed of single promoter-less genes (coding sequence, CDS), so they rely on $P_{C}$ promoter to drive their expression. Starting 
from $P_{C}$, their expression gradually decreases in the array (Jacquier et al., 2009) (Figure 1A). Then, the gene cassettes that are located close enough to the $\mathrm{P}_{C}$ promoter are the only ones to be expressed, except for which that contains their own promoter (Biskri and Mazel, 2003; da Fonseca and Vicente, 2012; Stokes and Hall, 1991; Szekeres et al., 2007). The integron system represents a low-cost and modular reservoir of adaptive functions for their host bacteria. Each gene cassette is flanked by an attC recombination site that is recognized and recombined by the integrase. The latter catalyzes the different reactions that lead to cassette mobilization. By recombining attI and attC sites, the integrase allows the recruitment of cassettes downstream of the $\mathrm{P}_{\mathrm{C}}$ promoter and express them (Figure $\left.1 \mathrm{~A}\right)$. By recombining two consecutive attC sites, the integrase ensures the excision of one cassette. Subsequent excision and integration of a circular cassette in the first position of the array, constitute a way for a previously silent gene to be expressed (Figure 1A).

A key feature of the integron is the ability of the integrase to recombine both single-stranded DNA (attC site) and double-stranded DNA (attI site) depending on their structure and sequence respectively (Figure 1B) (Bouvier et al., 2005; Bouvier et al., 2009). Indeed, each integrase recognizes the sequence of its cognate attI site (Biskri et al., 2005; Collis et al., 2002). In contrast, the integrase does not recognize the sequence of attC sites, but rather the structure of the ss folded bottom strand (bs) (Figure 1B) (Bouvier et al., 2005; Bouvier et al., 2009; Francia et al., 1999; Johansson et al., 2004). This specific recognition of the folded bottom strand of attC sites allows the insertion of cassettes in the proper orientation so that they can be expressed by the $\mathrm{P}_{\mathrm{C}}$ promoter (Bouvier et al., 2009; Nivina et al., 2016). The recognition of this specific ssDNA substrate imposes some constraints for recombination reactions. Indeed, during the attI $\times$ attC reaction, an atypical Holliday junction $(\mathrm{aHJ})$ is formed (Figure 1B), which cannot be resolved by the classical way but can be by a hostdependent replicative pathway (Loot et al., 2012). Several other host processes are implicated in cassette recombination, for instance, by influencing the proper folding of attC site (Loot et al., 2010; Loot et al., 2017). Host cells also control integrase and cassette expressions (Baharoglu et al., 2012; Krin et al., 2014; Strugeon et al., 2016). The most relevant regulatory network is the induction of integrase expression, for both Class $1 \mathrm{MI}$ and $V$. cholerae SCI systems, in response to environmental stress through the SOS response (Cambray et al., 2011; Guerin et al., 2009). Such regulation allows the conditional reshuffling of cassettes, at moment where the cells need to adapt to environmental changes. These examples show how integrons are intricate host-cell connected systems to maximize the potential benefit conveyed by this “adaptation on demand” device (Escudero et al., 2015). 
Until then, the recombination process occurring in MIs were largely studied and the majority of assays were developed and performed in E. coli strains. Paradoxically, our knowledge on the SCI of the $V$. cholerae pathogenic strain remain predominantly descriptive and this, more than 20 years after its discovery and despite being the paradigm in the field. Here, for the first time, we designed experimental assays to study cassette recruitment dynamic directly in the $V$. cholerae SCI. We delivered cassettes substrates using conjugation but also natural transformation since $V$. cholerae is known to be naturally competent and to exchange DNA by this way. We obtained significant cassette insertion rate mediated by the sole endogenous integrase. We confirmed that this integrase expression is due to the SOS activation probably triggered by the single-stranded cassette delivery during conjugation and transformation processes. Interestingly, cassettes are preferentially recruited into the attIA primary recombination site, directly downstream of the $\mathrm{P}_{\mathrm{C}}$ promoter, ensuring their expression and consecutive testing for selective advantages they can confer.

By performing in vivo recombination assays, we showed that RecA protein $\left(\operatorname{Rec} A_{V c h}\right)$ is critical for the cassette recruitment in the attIA site of the $V$. cholerae SCI. The impact of $\mathrm{Rec}_{\mathrm{Vch}}$ on cassette recombination is SOS-independent and seems specific of the $\operatorname{attIA} \times$ attC reaction mediated by the integrase of $V$. cholerae, IntIA. Indeed, the RecA $V_{c h}$ protein did not influence neither $a t t C \times a t t C$ recombination mediated by IntIA nor the MI recombination cassettes. Moreover, unlike that of MIs, the $V$. cholerae SCI is not active in others bacterial hosts (e.g., in E. coli, even supplemented with the RecAvch). Altogether, these results suggest that, in contrast of MIs, some specific host factors can regulate cassette recombination in SCIs. Therefore, MIs might have evolved from the SCIs by overcoming some host factors. In addition to their association with transposons and conjugative plasmids, this evolutionary trait may explain the large MI dissemination among bacteria and the antibioresistance expansion.

\section{MATERIAL AND METHODS}

\section{Bacterial strains and media}

E. coli and V. cholerae strains were cultivated at $37^{\circ} \mathrm{C}$ in Luria Bertani (LB). V. cholerae and E. coli strains, which contain a plasmid with a thermo-sensible origin of replication were grown at $30^{\circ} \mathrm{C}$. Thymidine (Thy) and diaminopimelic acid (DAP) were supplemented, when necessary, to a final concentration of $0.3 \mathrm{mM}$. Glucose (Glu), L-arabinose (Ara) and isopropyl- $\beta$-D-thiogalactopyranoside (IPTG) were added in media respectively at final concentration of $10,2 \mathrm{mg} / \mathrm{ml}$ and $0.8 \mathrm{mM}$. To induce $\mathrm{P}_{\text {tet }}$ promoter anhydrotetracycline (aTc) 
was supplemented in media to a final concentration of $1 \mu \mathrm{g} / \mathrm{ml}$. In the case of E. coli strains, antibiotics were added at the following concentrations: carbenicillin (Carb), $100 \mu \mathrm{g} / \mathrm{ml}$, chloramphenicol (Cm), $25 \mu \mathrm{g} / \mathrm{ml}$, kanamycin (Km), spectinomycin (Sp), $50 \mu \mathrm{g} / \mathrm{ml} . \quad V$. cholerae strains were cultivated with the same antibiotic concentrations except in the case of $\mathrm{Cm}$ and Sp, that were supplemented at a final concentration of $5 \mu \mathrm{g} / \mathrm{ml}$ and $100 \mu \mathrm{g} / \mathrm{ml}$ respectively. When $V$. cholerae strains were cultivated in presence of glucose, the later concentration of Sp was increased 2-fold (200 $\mu \mathrm{g} / \mathrm{ml})$.

\section{Plasmid and strain construction}

The different plasmids and strains that were used in this study are described in Table S1 and S2. Primers used to construct the different vectors are listed in Table S3.

We performed allelic exchange to construct N16961 $\Delta r e c A$, N16961 $\Delta r e c A \Delta a t t I A$, N16961 ArecA AattIA::attI1 and N16961 hapR+ lexA(ind-). To this purpose, we used different variants of the pMP7 vector, respectively pB203, pK590, pK584 and p6780 (Guerin et al., 2009). We followed the same protocols as previously described (Le Roux et al., 2007; Val et al., 2012). Briefly, the suicide vector pMP7 contains a R6K origin of replication and its replication is then dependent on the presence of the $\Pi$ protein in the host cell. The $\Pi 3813$ cell, a pir + CcdB resistant E. coli strain (Le Roux et al., 2007), was used for cloning the different pMP7 plasmids. Once constructed, these vectors were transformed into the $\beta 3914$ donor strain (Le Roux et al., 2007) in order to deliver by conjugation the pMP7 vector into the desired recipient strain. Homology regions corresponding to the genomic DNA from the recipient strain have been cloned in the different pMP7 vector to allow the integration of the plasmid by homologous recombination. The only way for pMP7 vector to replicate into recipient strains is then to integrate into the host genome after a first crossover. After conjugation, integration of the entire pMP7 vector were then selected by plating cells on Cm plates lacking DAP. Next, cells were grown in presence of L-arabinose $(0.2 \%)$ in order to express the CcdB toxin. The expression of this toxin allows to kill cells in which the second crossover that leads to the excision of pMP7 backbone did not take place. This method allows us to obtain mutants of $V$. cholerae that are devoid of any antibiotic resistance marker. Note that for the deletion or replacement of attIA site in N16961 $\Delta r e c A$ strains, we previously transformed this cell with pAM::rec $A_{E c}$ vector (pCY579 (Cronan, 2003). The expression of $\operatorname{Rec}_{E c}$ protein allows allelic replacement to take place in N16961 $\Delta r e c A$ mutant. At the end of construction, strains were cultivated without Carb and IPTG and loss of the pAM::rec $A_{E c}$ 
plasmid was assessed. For ectopic complementation of the recA mutation, we insert a copy of the $\operatorname{rec} A$ gene into the attTn7 site, which is present on the chromosome of $E$. coli and $V$. cholerae. We used the same strategy as the one described in (de Lemos Martins et al., 2018). The helper plasmid pMVM1 was transformed into both N16961 and MG1655 recA mutants. This vector has a thermo-sensitive origin of replication and carries a $\mathrm{P}_{\mathrm{BAD}}$ promoter that triggers the expression of TnsABCD transposases. These transposases catalyze insertion into attTn7 at high frequency. A second shuttle vector, pMP234, carries the IR sites that are recognized by the transposases and was modified for specific integration of the recA gene from E. coli or $V$. cholerae. The FRT-aph-FRT cassette was also added in between the IR, in order to select for transposition event. The pMP234 vector is a derivative of the pSW23T suicide vector, so its replication cannot take place into recipient cells that lack the $\Pi$ protein. For integration, the pMP234 shuttle vector was delivered by conjugation in the recipient strains containing pMVM1. Transposition events were selected by plating conjugants on $\mathrm{Km}$ plates without DAP. These plates were incubated overnight at $42^{\circ} \mathrm{C}$ to get rid of the helper vector. The integration of $\mathrm{P}_{\mathrm{LAC}}$-recA-FRT-aph-FRT fragment was assessed by testing UV sensitivity of the strains and by performing PCR and subsequent sequencing. After integration, the Flippase (Flp) expressing vector (pMP108, Carb ${ }^{\mathrm{R}}$ (de Lemos Martins et al., 2018)) was delivered by conjugation into the $V$. cholerae strain in order to excise the Km resistance cassette. This plasmid is easily lost when culturing $V$. cholerae strains without Carb. In the case of E. coli, we transformed the strains with the pCP20 Flp expressing vector (Carb $^{\mathrm{R}}$ (Cherepanov and Wackernagel, 1995)), which has a thermo-sensitive origin replication.

\section{Suicide conjugation assay}

This assay has been previously described (Bouvier et al., 2005) and implemented (Biskri et al., 2005) for the delivery of one specific strand of a recombination site into recipient strains that express the integrase. In this study, we used the suicide conjugative vector pSW23T (pD060) that allows the delivery of the bottom strand of the $a t t C_{a a d A 7}$ recombination site. This vector carries a RP4 origin of transfer and an ori $V_{\mathrm{R} 6 \mathrm{~K} \gamma}$ origin of replication. It was previously transformed into a pir+ donor strain, $\beta 2163$, which contains the RP4 machinery of transfer. This later strain needs DAP to grow in rich medium, which allows its counter-selection after that conjugation took place. The only possibility for pSW23T to replicate into the pirrecipient strains is then to insert into the genome through a recombination reaction catalyzed by the IntI protein. Since the pSW23T vector contains a $\mathrm{Cm}^{\mathrm{R}}$ cassette, recombination events 
can be selected with this marker. By plating in parallel conjugants on solid media that contain or not $\mathrm{Cm}$, we are able to establish the frequency of a given recombination reaction. We adapted this protocol for the use of $V$. cholerae as recipient strain, in which plasmids are more easily lost in absence of antibiotic selection than in E. coli. In this case, after an overnight culture, recipient cells were diluted (1:100) and grown in presence of Sp and Ara (0.2\%) respectively to maintain pBAD43 vector and to allow the expression of the integrase. In the case of the $r e c A_{V c h}$ and $r e c A_{E c}$ complemented strain, ITPG was also added in the media. The donor strain was grown in parallel in presence of DAP. When both donor and recipient cultures reach an $\mathrm{OD}_{600 \mathrm{~nm}}$ of $0.6,1 \mathrm{ml}$ of each culture was harvested by centrifuging 6 min at $6000 \mathrm{rpm}$. The obtained pellet was re-suspended in a droplet of LB and spread on a $0.45 \mu \mathrm{m}$ filter. This filter was placed on MH DAP, Ara plates and incubated at $37^{\circ} \mathrm{C}$ during $3 \mathrm{~h}$. After incubation, the filter was re-suspended in $5 \mathrm{ml}$ of LB and this suspension was used to spread appropriate dilutions on $\mathrm{MH}, \mathrm{Cm}$, Sp, Glu and $\mathrm{MH}$, Sp, Glu plates. After 2 days of incubation at $37^{\circ} \mathrm{C}$, the recombination frequency was calculated as the ratio of $\mathrm{Cm}^{\mathrm{R}}$ clones over the total number of recipient colonies that grew on $\mathrm{MH}$, Sp, Glu plates. Note that in the case where we did not detect recombination event for one replicate, we calculate the recombination rates as the ratio of the mean of recombinant clones over the mean of total recipient clones obtained for the different replicates. In this case no error bars are represented on our graphics.

\section{Integrase mediated recombination after natural transformation}

In this study, we used the same pSW23T vector (pD060) that used in the suicide conjugation assay. V. cholerae natural transformation was previously described (Marvig and Blokesch, 2010). Here, we adapted this protocol to assess integrase-mediated recombination frequency after natural transformation in naturally competent strains (hap $\left.{ }^{+}\right)$of $V$. cholerae. An overnight culture was used to inoculate 1:100 of bacteria in $5 \mathrm{ml}$ of LB medium supplemented with Sp $(50 \mu \mathrm{g} / \mathrm{ml})$. The culture was grown at $30^{\circ} \mathrm{C}$ until they reached an $\mathrm{OD}_{600 \mathrm{~nm}}$ of 0.5 . One milliliter of cells was then centrifuged (5000 rpm, 10 minutes) and resuspended in $1 \mathrm{ml}$ of M9 minimal medium supplemented with $\mathrm{MgSO} 4(32 \mathrm{mM})$ and $\mathrm{CaCl}_{2}(5 \mathrm{mM})$ and $\mathrm{Sp}(100$ $\mu \mathrm{g} / \mathrm{ml}$ ). Tubes containing 50 to $80 \mathrm{mg}$ of chitin (C9213; Sigma) were inoculated with $0.5 \mathrm{ml}$ of washed cells and $0.5 \mathrm{ml}$ of fresh M9 medium supplemented with $\mathrm{MgSO}_{4}, \mathrm{CaCl}_{2}$ and $\mathrm{Sp}$, vortexed, and grown 48 hours at $30^{\circ} \mathrm{C}$ with shaking. Cultures were then washed (5000 rpm, 10 minutes) and resuspended in an equal volume of fresh M9 medium supplemented with MgSO4, $\mathrm{CaCl}_{2}$, Sp and anhydrotetracycline (aTc) to induce integrase expression. The cultures 
were incubated again for 30 minutes at $30^{\circ} \mathrm{C}$ with shaking. Then, $2 \mu \mathrm{g}$ of plasmid DNA (the pD060 vector) were added to the cultures and incubated for $36 \mathrm{~h}$ at $30^{\circ} \mathrm{C}$ with shaking. After incubation, bacteria were detached from chitin by vortexing vigorously during 30 seconds. The obtained bacteria suspension was used to spread appropriate dilutions on $\mathrm{MH}, \mathrm{Cm}$, Sp and $\mathrm{MH}$, Sp plates. After 2 days of incubation at $37^{\circ} \mathrm{C}$, the recombination frequency was calculated as the ratio of $\mathrm{Cm}^{\mathrm{R}}$ clones over the total number of recipient colonies that grew on $\mathrm{MH}$, Sp plates in the same manner as for suicide conjugation assay.

\section{Analysis of cassette insertion point localization}

For each experiment, at least eight recombinant clones were isolated on appropriate plates and analyzed by PCR. For this, we performed different PCR reactions. In order to determine precisely if the pSW23T vector has been inserted into the attIA site of the SCI we used 5778 and SWend primers. These primers hybridize respectively in a sequence upstream of attIA in $V$. cholerae chromosome 2 or downstream of att $C_{a a d A 7}$ in the pSW23T vector. For E. coli and $V$. cholerae strains transformed with the pSU38A or on pBAD43 vectors that harbor recombination sites, SWbeg and MFD or 1704 and SWend primers respectively were used to amplify one junction of the co-integrate. Finally, to detect insertion of pSW23T in the genome of V. cholerae, either at secondary sites or into the VCR sites of the SCI, we performed random PCR amplification. For these, we performed a first random PCR reaction using the 1863 degenerated and the 2405 primers. The 2405 primer hybridizes upstream of the attC sites on pSW23T plasmids. Due to the presence of degenerate nucleotides in the 1863 primer, low hybridization temperatures were used, first, $30^{\circ} \mathrm{C}$ during 5 cycles and after, $40^{\circ}$ during 30 cycles. The obtained amplified DNA fragments were subjected to a second PCR reaction in order to enrich for PCR products corresponding to cassette insertion. For this purpose, we used 1865 and 1388 primers. These primers hybridize respectively to the fixed part of the degenerated 1863 primer and upstream (but closer than 2405) of the attC sites on pSW23T plasmids. Recombination points were precisely determined by sequencing PCR products using 1366. The 1366 primer hybridizes upstream (but closer than 1388) of the attC sites on pSW23T plasmids. In all cases, some PCR reactions were purified and sequenced to confirm the insertion point.

\section{Recombination assay with unidirectional replicative substrate}

This assay was previously described (Loot et al., 2010) and allow the determination of the recombination rates of a given recombination reaction when attC sites are carried by a 
replicative plasmid. The vectors that we used (p7523 or p7546, $\mathrm{Cm}^{\mathrm{R}}$ ) replicate unidirectionally and the $a t t C_{a a d A 7}$ recombination sites they carry have been cloned, so that the recombinogenic bottom strand is located either on leading or lagging strand template ("lead" or "lag" orientation). V. cholerae strains were transformed with these unidirectionalreplicative vectors and the IntIA expressing vector $\left(\mathrm{p} 995, \mathrm{Carb}^{\mathrm{R}}\right.$ ) or with the empty version of the plasmid (p979, $\mathrm{Carb}^{\mathrm{R}}$ ). As p7523 and p7546 have both a thermo-sensitive origin of replication, the cells were cultivated overnight at $30^{\circ} \mathrm{C}$. The next day, cultures were diluted (1:100) and incubated during $5 \mathrm{~h}$ at $30^{\circ} \mathrm{C}$ in presence of $\mathrm{Cm}$ and Carb to maintain both pTSC29 and pBAD18 plasmid. In order that recombination takes place, L-arabinose was also added in the media at a concentration of $0.2 \%$ to allow the expression of the integrase. After 5h of incubation, appropriate dilutions of the cultures were plated in parallel on $\mathrm{MH} \mathrm{Cm}$, Carb, Glu and MH Carb, Glu plates and incubated two days at $42^{\circ} \mathrm{C}$. Since pTSC29 vector cannot replicate at $42^{\circ} \mathrm{C}$, the selection on Cm plates at this temperature allows recovering only the clones in which att $C_{a a d A 7}$ site have been recombined by IntIA. Recombination rates were calculated as for suicide conjugation assays, by considering the ratio of $\mathrm{Cm}^{\mathrm{R}}$ clones over total number of recipient clones that grew on MH Carb, Glu plates. In order to determine if cassette insertion occurs into the attIA site of the SCI of $V$. cholerae, we performed PCR reactions on at least eight random isolated clones using 5778 and 573 or 5778 and MFD primers (respectively used for "lead" and "lag” orientation of the bs of att $C_{a a d A 7}$ site on pTSC vector).

\section{RESULTS}

Horizontally transferred cassettes are efficiently inserted into the attIA site of the Vibrio cholerae $S C I$

Large SCIs such as the $V$. cholerae SCI constitute huge reservoirs of functions for their host bacteria. In such large integrons, several recombination sites can constitute potential targets for cassette insertion (attIA primary recombination site and/or the numerous VCR sites). In a previous study, recombination assays that were performed in $V$. cholerae aimed at evaluating cassette insertion in recombination sites carried on plasmids (Biskri et al., 2005). Here, we attempt to visualize cassette insertion into the SCI of $V$. cholerae using our classical conjugation assay (Bouvier et al., 2005) and developing a natural transformation assay. Interestingly, both assays reproduce the natural conditions in which the acquisition of cassettes could occur through horizontal gene transfer in $V$. cholerae (Figure 2A). The 
plasmids containing the donor attC site (pSW23T::att $\left.C_{a a d A 7}\right)$ are delivered on a singlestranded form in a recipient $V$. cholerae strain containing a vector expressing the IntIA integrase. Once delivered, attC-containing plasmids cannot replicate and can therefore be assimilated to a non-replicative integron cassette. The unique way for these synthetic cassettes to be maintained is to be inserted in the $V$. cholerae host genome by a recombination between the cassette $a t t C$ sites and the SCI att sites.

When we carried out this test by delivering cassette by conjugation assay, we detected a significant level of insertion $\left(7.5 \times 10^{-3}\right)$ of the pSW23T::attCaadA7 vector using the N16961 $w t$ V. cholerae strain (Figure 2B, + IntIA). We performed PCR reactions on some recombined clones and demonstrated that, in all cases, the cassette insertion occurs at attIA sites from $V$. cholerae SCI platform (Figure S1A). By sequencing some PCR products, we confirmed that insertion point was correctly localized in the 5'-AAC-3' triplet.

We took advantage from $V$. cholerae natural competent state in the presence of chitin (Meibom et al., 2005) to investigate for cassette recombination in the context of another HGT mode. We adapted natural transformation protocol to evaluate cassette insertion frequency in the $V$. cholerae SCI (Figure 2A). We obtained a recombination frequency of $4.8 \times 10^{-7}$ when overexpressing the integrase (Figure 2B, +IntIA). Importantly, these recombination frequencies, which are much lower than those obtained during conjugation in the same conditions (integrase overexpression), are the consequences of a limited proper frequency of natural transformation (Marvig and Blokesch, 2010). We also performed PCR reactions on some recombined products and demonstrated that cassette insertion occurs, for all tested clones, at attIA sites from $V$. cholerae SCI platform (Figure S1B). These results show that, during conjugation or natural transformation, integron cassettes are efficiently released in the $V$. cholerae host cell and inserted in the SCI. Interestingly, the large majority of insertion events occurs in the integron platform attIA in spite of the presence of 180 attC sites meaning that all newly inserted cassettes are expressed and therefore tested for their selective advantage.

The endogenous integrase efficiently inserts cassettes into the attIA site of the Vibrio cholerae $S C I$

We also performed the both assays in the presence of the sole endogenous SCI IntIA integrase. Interestingly, in this case, we also detected a significant level of insertion of the pSW23T::att $C_{a a d A 7}$ vector using the N16961 wt V. cholerae strain $\left(1.8 \times 10^{-6}\right.$ and $6.1 \times 10^{-7}$ respectively for conjugation and transformation assays, Figure 2B, -IntIA). Here again, we 
performed PCR reactions on some recombined products and demonstrated that cassette insertion occurs, for all tested clones, at attIA sites from $V$. cholerae SCI platform. This recombination activity is due to the expression of the endogenous intIA integrase gene since no recombination event was detected in the strain devoid of endogenous integrase (N16961 $\triangle$ intIA) for both assays (Figure 2B, -IntIA). We also demonstrated that the expression of endogenous integrase is dependant of the SOS system since no recombination event was detected below the detection limit of $1.5 \times 10^{-8}$ in the N16961 lexAind- strain in which the SOS response is not inducible. Indeed, in this strain, the SOS regulon genes are constitutively repressed because of the presence of the uncleavable LexA $A_{\text {A91D }}$ version of the LexA repressor (Guerin et al., 2009). As supplementary control, we also overexpressed the IntIA integrase in these both mutant strains and as expected we obtained a very high recombination frequency (respectively $5.4 \times 10^{-3}$ and $6.0 \times 10^{-3}$ in the $\Delta i n t I A$ and lexAind- strains, Figure 2B, + IntIA). Altogether, these results show that, during conjugation or natural transformation, integron cassettes are efficiently released in the $V$. cholerae host cell and inserted in the SCI even in the presence of the sole endogenous integrase. The level of endogenous integrase expression, triggered by SOS response induction initiated by the single-stranded cassette entry during conjugation and natural transformation (Baharoglu et al., 2010) seems sufficient to insert cassettes at significant level in the $V$. cholerae SCI. Note that, when performing both conjugation and natural transformation, we detected some attIA insertion events associated with shuffling of cassettes in first position (1/160 and 12/152 respectively, Figure S1).

\section{$\operatorname{Rec}_{V c h}$ influences cassette insertion into the attIA site of the Vibrio cholerae SCI}

Here, we precisely investigated the cassette recombination mechanism used by the SCI of $V$. cholerae. To define the network of intervening host factors, we tested the effect of the Vibrio cholerae RecA protein $\left(\mathrm{Rec}_{\mathrm{Vch}}\right)$. The RecA protein is functionally conserved among bacterial species (Goldberg and Mekalanos, 1986) and in eukaryotic organisms (Shinohara and Ogawa, 1999). RecA is a critical enzyme for homologous recombination process, during which it binds ssDNA catalyzing the pairing with complementary regions of dsDNA and strand exchange reactions (Cox, 2007a; Kowalczykowski, 2000; Lusetti and Cox, 2002). Because of the capacity of the RecA protein to bind ssDNA, we tested its impact on SCI cassette recombination catalyzed by IntIA in $V$. cholerae. Among the several conditions previously developed, we chose to use the optimal one, i.e., the suicide conjugation assay with overexpression of integrase (Figure 3A). Here again, we obtained a very high recombination rate $\left(2.0 \times 10^{-2}\right)$ when using the wt parental strain as 
recipient strain. We observed a decrease of more than two orders of magnitude in the recombination rates $\left(7.7 \times 10^{-5}\right)$ in the corresponding N16961 $\Delta r e c A$ mutant strain (Figure 3A). When performing PCR analysis for each reaction, we confirmed that insertions occur in the attIA site (222/222 and 108/111, respectively for the $w t$ and $\Delta r e c A$ strains). These results mean that the RecA ${ }_{V c h}$ protein favors insertion of cassettes in the attIA site of $V$. cholerae SCI. As expected, no recombination event was detected in the N16961 $\triangle \mathrm{rec} A$ control strain that carries the empty pBAD43 vector (compare to the N16961 wt strain, Figure 3A), since the SOS response cannot be activated and induce the endogenous integrase expression in these cells. To confirm that the decrease in recombination rates that we observed is specifically due to the deletion of the $r e c A_{V c h}$ gene and not to polar effects, we constructed a strain in which the $r e c A_{V c h}$ deletion was ectopically complemented. For this, a copy of the $r e c A_{V c h}$ gene was inserted into the att $\operatorname{Tn} 7$ site located in the chromosome 1 of $V$. cholerae (de Lemos Martins et al., 2018). The $r e c A_{V c h}$ ectopic complementation allows recovering the same recombination rate as in the N16961 wt strain (Figure 3A), meaning that the effect on attIA $\times$ attC $C_{a a d A 7}$ recombination reaction, which we observed, is specific to the absence of the RecA $V_{v c h}$ protein. Furthermore, we tested the ability of the RecA protein of $E$. coli $\left(\operatorname{Rec} A_{E c}\right)$ to complement the $r e c A_{V c h}$ deletion. The RecA protein of $E$. coli and $V$. cholerae show variations in the amino acid sequence of their respective C-terminal part (Figure S2). This C-terminal region is implicated in the modulation of $\operatorname{Rec}_{E c}$ activity notably by interacting with regulator proteins (Cox, 2007b; Lusetti et al., 2004). We then tested if such variations could lead to host specific regulation that will impact cassette recombination. For this, we inserted a copy of the recA gene of $E$. coli into the attTn7 locus in $V$. cholerae. We therefore demonstrated that the complementation of the $\mathrm{N} 16961 \Delta \mathrm{rec} A$ mutant with $\mathrm{Rec}_{E c}$ was as efficient as with the native Rec $_{V c h}$ of $V$. cholerae (Figure $3 \mathrm{~A}$ ). This is consistent with the high percentage of identity (almost $80 \%$, Figure S2) shared by both RecA proteins. Moreover, we observed that the expression of Rec $A_{E c}$ also triggers SOS induction leading to endogenous integrase expression in the control strain harboring the empty pBAD43 plasmid (Figure 3A). Both RecA proteins seem then to share enough identity so that the $\operatorname{Rec}_{E c}$ can replace Rec $A_{V c h}$ for several functions including its apparent role in cassette recombination in V. cholerae.

Since RecA is involved in the induction of the SOS response, its effect on cassette recombination could be indirect and involve one or more proteins of the SOS regulon. Yet, we previously demonstrated that into the lexAind- mutant, in which the SOS response is noninducible, we did not observe any effect on recombination efficiency compare to the $w t$ strain (Figure 2B). The use of this strain allows us to uncouple the effect on SOS induction from 
other properties of the RecA protein and to demonstrate that the RecA $v_{v c}$ effect is not due to the SOS regulon proteins. We also determined the role of the Rec $\mathrm{A}_{V c h}$ protein during cassette recombination in attIA when this site is moved on a plasmid (Figure 3A). For this, we constructed a $V$. cholerae strain deleted for the resident attIA site ( $V$. cholerae N16961 $\triangle a t t I A)$ and transformed with an attIA-containing plasmid. Once again, we obtained a decrease of two orders of magnitude (from $2.0 \times 10^{-2}$ to $2.4 \times 10^{-4}$, Figure 3B).

Together these results indicate that the RecA protein of $V$. cholerae favors the attIA $\times$ attC recombination mediated by IntIA, in a SOS-independent manner and whatever the attIA localization.

\section{RecAvch does not influence attC $\times$ attC recombination in Vibrio cholerae}

During both $a t t C \times a t t C$ and $a t t I \times a t t C$ reactions, the proper folding of attC site is required for binding of integrase monomer. If the RecA protein acts on cassette recombination through its ssDNA binding capacity affecting attC site folding, we expect that it will impact both attI $\times a t t C$ and $a t t C \times a t t C$ reactions that are catalyzed by IntIA. To test this, we used different assays that allow to assess cassette insertion frequencies directly into the VCR sites of the SCI or in attC sites carried on plasmids (Figure 4). To observe insertion events into the VCR sites of the SCI, we used the $V$. cholerae mutant strain devoid of the resident attIA site (V. cholerae N16961 $\triangle a t t I A$, see just above). Here, we did not observe any effect of the RecA protein in the recombination rates obtained with $\triangle a t t I A$ and $\triangle a t t I A \Delta r e c A_{V c h}$ mutant strains. To confirm the cassette insertion in VCRs, we performed random PCR reactions (Material and methods). The recombination rates into VCR sites is similar between both mutant strains (Figure 4A) meaning that the Rec $A_{V c h}$ protein does not affect the $a t t C \times a t t C$ recombination.

To confirm this absence of Rec $A_{V c h}$ protein effect on $a t t C \times a t t C$ recombination, we perform a second conjugation assay in $\mathrm{V}$. cholerae N16961 $\triangle$ attIA strains transformed with a plasmid carrying the MI attC $C_{a d A 1}$ site (from the aadA1 gene cassette). We obtained a significant rate of recombination for both $\triangle a t t I A$ and $\triangle a t t I A \Delta r e c A_{V c h}$ strains and we did not observe any impact of the RecA $A_{V c h}$ protein on $a t t C_{a a d A 1} \times a_{t t} C_{a a d A 7}$ reaction catalyzed by IntIA in $V$. cholerae (Figure 4B). Together, these results, with results presented in the last paragraph, indicate that the $\mathrm{Rec}_{V c h}$ protein seems to favor only attIA $\times$ attC reaction and not to influence insertion reactions into attC sites, whether they are present on the chromosome (VCR) or on a plasmid (MI attC sites). 


\section{Rec $_{V c h}$ does not influence attI1 $\times$ attC mediated by IntI1 in Vibrio cholerae}

The previously demonstrated influence of the Rec $A_{V c h}$ protein on cassette recombination was striking since it was already demonstrated that the Rec $A_{E c}$ protein does not influence the recombination reactions catalyzed by IntI1 in E. coli (Loot et al., 2014). To determine if the role of the RecA $A_{V c h}$ protein is specifically linked to V. cholerae SCI system, we used two different recombination tests to establish, in $V$. cholerae, the activity of the integrase IntI1. First, we performed the suicide conjugation assays in a $V$. cholerae strain where the attIA site from the SCI platform was replaced by the attI1 site (the cognate site of the IntI1 integrase). Secondly, we performed this assay in $V$. cholerae strains deleted for the attIA site (V. cholerae N16961 $\triangle a t t I A)$ and transformed with an attI1-carrying plasmid. We observed, using these two assays, that the frequencies of attI1 $\times$ attC reaction catalyzed by IntI1 were the same for both $\triangle a t t I A$ and $\triangle a t t I A \triangle r e c A$ recipient strains (Figure 5A and 5B). Therefore, we demonstrated that, in $V$. cholerae, the attI1 $\times$ attC reaction catalyzed by IntI1 is not influenced by Rec $A_{V c h}$. This suggests that the Rec $A_{V c h}$ influence of the $a t t I \times a t t C$ reaction is specific to IntIA.

\section{Effect of RecA on cassette recombination in Escherichia coli}

As already introduced, the impact of the RecA protein on cassette recombination was already tested in E. coli (Loot et al., 2014). In our previous study, a setup based on the reconstitution of a functional dapA gene after cassette excision through an intramolecular reaction was used. In this case, only the efficiency of reactions catalyzed by the integrase of Class 1 MI was studied (Loot et al., 2014). Here, we assessed the efficiency of either attI $\times$ attC or attC $\times$ attC intermolecular reactions and we tested the effect of $r e c A$ deletion on reactions catalyzed by both IntI1 and IntIA integrases. We also used another attC site, VCRVCA0441 (Loot et al., 2014). We still observed the RecA-independence of IntI1 for both attI1 $\times$ attC $C_{a a d A 7}$ and VCR $\times$ $a t t C_{a a d A 7}$ reactions (Figure $6 \mathrm{~A}$ and $6 \mathrm{~B}$, left panels). Indeed, respective recombination frequencies were identical in MG1655 wt and $\triangle r e c A$ recipient strains. For the reaction catalyzed by IntIA, we observed that, in $E$. coli, the VCR $\times$ attCaadA7 reaction was not affected by the deletion of the recA gene (Figure 6B, right panel). In accordance with the study of Biskri and coll. (Biskri et al., 2005), we found that IntIA $V_{c h}$ was not able to efficiently catalyze the attIA $\times$ att $C_{a a d A 7}$ reaction in $E$. coli in the $w t$ strain (Figure 6A, right panel). As proposed earlier, if IntIA recombines much less efficiently in E. coli this may reflect the absence, or a too large divergence, of at least one host factor required for this reaction. Thus, in order to determine if RecA could correspond to one of these divergent factors, we tested if 
Rec $_{V c h}$ could rescue the lack of attIA $\times$ attC recombination in E. coli. We inserted, as performed previously in $V$. cholerae, the $r e c A_{V c h}$ gene into the unique att $\operatorname{Tn} 7$ site of $E$. coli. However, we found that the expression of RecA ${ }_{V c h}$ do not restore the recombination capacity of IntIA for the attIA $\times$ att $C_{a a d A 7}$ reaction in E. coli. Thus, it shows that the RecA protein is not the factor whose divergence hamper the $a t t I A \times a t t C_{a a d A 7}$ reaction catalyzed by IntIA to take place in E. coli.

\section{RecAvch influences attIA cassette insertion however the attC site is delivered}

485 In all previously presented assays, we used horizontal gene transfer mechanisms as a mean for delivering the single-stranded recombinogenic bottom strand of the attC site on nonreplicative substrate mimicking integron cassettes. To determine if RecA still favors cassette insertion in the case where $a t t C$ sites are folded from double-stranded molecules, we performed a recombination assay that relies on the use of replicative vectors (pTSC29 derivatives). These vectors replicate unidirectionally and the att $C_{a a d A 7}$ site that they carry was cloned in two orientations, so that the bottom strand of the attC site is either present on lagging or leading strand template (Figure 7A). When the bs of attC site is located on the lagging strand template, in which large region of ssDNA are available during replication (i.e., between Okazaki fragments), bs attC site folding from ssDNA is favored (Figure 7B). When bs of the attC site is located on the leading strand template, bs attC site folding can only occur from dsDNA, by cruciform structures extrusion (Loot et al., 2010) (Figure 7B). Since the pTSC29 vectors have a thermo-sensitive origin of replication, the selection of recombinant $\mathrm{Cm}^{\mathrm{R}}$ clones at $42^{\circ} \mathrm{C}$, allows us to evaluate the efficiency of recombination events. When we performed this replicative assay in the $V$. cholerae N16961 wt strain, we observed a decrease of about one order of magnitude when the att $C_{a a d A 7}$ bs is present on the leading strand template (Figure 7C). Such slight differences in the recombination frequency between the two orientations of the $a t t C_{a a d A 7}$ site were previously observed, when the same recombination assays were carried out in E. coli with the integrase IntI1 (Loot et al., 2010). Concerning the N16961 $\triangle \mathrm{rec} A$ mutant, we still observed a large decrease in recombination rates for both $a_{t t C_{a a d A 7}}$ site orientations (Figure 7C). These results demonstrate that Rec $\mathrm{A}_{V c h}$ is involved in the mechanism of recombination during attIA $\times a t c$ reaction whatever the manner that the $a t t C$ site is delivered (i.e., from ssDNA or dsDNA). 


\section{DISCUSSION}

512

513

514

515

516

517

518

519

520

521

522

523

524

525

526

527

528

529

530

531

532

533

534

535

536

537

538

539

540

541

542

543

Incoming integron cassettes are efficiently inserted in the attIA site of the Vibrio cholerae integron

Until now, integron cassette movements in natural integrons, mediated by the endogenous integrase, have never been experimentally demonstrated. Indeed, exchange of gene cassettes between bacterial species (Domingues et al., 2012) and gene cassette movements in the $V$. cholerae SCI (Baharoglu et al., 2010) have been detected but at very low frequencies and related to homologous recombination events. Here, for the first time, we worked with the entire endogenous SCI of the Vibrio cholerae pathogen strain and we succeed to visualize cassette recombination events dependent of the sole endogenous integron integrase. We used, to deliver the integron cassettes, both conjugation and natural transformation processes. These two frequent mechanisms perfectly mimic the natural conditions in which cassettes can be delivered through horizontal gene transfer in the Vibrio cholerae strains. When delivering cassettes by conjugation assay, we detected high rate of cassette insertion $\left(\sim 10^{-2}\right)$ in conditions where IntIA is overexpressed. However, we were also able to detect a significant level of cassette insertion $\left(>10^{-6}\right)$ catalyzed by the sole endogenous integrase expression. We also detect cassette insertion events catalyzed by the endogenous integrase by delivering cassette during natural transformation. The entry of single-stranded cassettes by natural gene transfer processes activates the SOS response sufficiently to express the endogenous integrase leading to the proper insertion of these incoming cassettes. We therefore reproduce the whole natural integron pathway in which incoming single-stranded integron cassettes by HGT processes activates their proper insertion in the integron platform (Figure 8).

Interestingly, by analyzing the insertion events, we demonstrated that despite the presence of 180 VCR sites in the large array of cassettes, the attIA site constitutes the major recruitment site for cassette insertion in the $V$. cholerae SCI. This recruitment, in the first position of the cassette array, ensures to the newly inserted cassettes to be efficiently expressed. This meaning that, even if the frequency of cassette insertion seems low when mediated by the endogenous integrase $\left(\sim 10^{-6}\right)$, these events can be selected and evolutionary fixed if cassette expression leads to an advantageous new function, which ensures an adaptive response to one environmental selective pressure. Thus, the knowledge of the precise cassette functions in SCI would allow to trace back the environmental conditions of cell growth and the evolutionary history of SCI-containing cells. 
545 Previous studies have shown how integrons are host-cell integrated systems. For instance, the folding of single-stranded attC substrates depends on several cellular processes including conjugation, replication or DNA topology (Loot et al., 2010; Loot et al., 2017). attC site folding can also be modulated by the binding of the SSB host protein, which, by hampering attC hairpin folding in vivo in the absence of IntI, would play an important role in maintaining the attC integrity and its recombinogenic functionality (Loot et al., 2014). It has also been shown that the pathway that allows the resolution of aHJ formed during attI $\times$ attC reaction is linked to a host-replicative process (Loot et al., 2012). Moreover, integrase expression is also extensively regulated by environmental stresses, thus connecting cassette recombination to the host-cell environment. Both integrase and cassette expressions, ensured respectively by $\mathrm{P}_{\text {int }}$ and $\mathrm{P}_{\mathrm{C}}$, are regulated by catabolite repression in V. cholerae SCI (Baharoglu et al., 2012; Krin et al., 2014). Expression of the MI class 1 integrase is in part regulated by the stringent response in biofilms (Strugeon et al., 2016). The most relevant example among these regulatory pathways, is undoubtedly the induction of integrase expression (of class $1 \mathrm{MI}$ and $V$. cholerae SCI) during the SOS response. Such regulation is ensured by the binding of the LexA repressor in the promoter region of $\mathrm{P}_{\text {int }}$ (Cambray et al., 2011; Guerin et al., 2009). Integrase expression like for all the other genes of the SOS regulon (more than 40 SOS genes), is unclenched by the autocatalytic cleavage of LexA, a process induced by the binding of RecA on single-stranded DNA constituting nucleofilament. This triggered SOS response is a global response to DNA damage ensuring a state of high-activity DNA repair. In this study, we investigated the role of the RecA protein in another regulatory point of the integron system, the cassette recombination. We choose to study this protein for two reasons. First, because of its ssDNA binding properties, since we know that cassette recombination involves one of the recombination sites, the $a t t C$ site, in a single-stranded DNA form. Second, because efficient cassette recombination could be dependent on one or several proteins belonging to the SOS regulon and whose expression is induced in presence of RecA. We found that the $\operatorname{Rec}_{V c h}$ protein is critical for the attIA $\times$ attC reaction in the SCI of $V$. cholerae. On contrary, when we tested the effect of Rec $A_{V c h}$ on cassette insertions in VCR sites of the SCI, we found that these $a t t C \times$ VCR reactions were independent of the RecA ${ }_{V c h}$ protein. We also confirmed the absence of RecA effect on $a t t C \times a t t C$ recombination by testing cassette insertion in an $a t t C_{a a d A 1}$ site-carrying plasmid. Interestingly, these results show that the RecA effect depend on the nature of att sites ( $a t t I$ or $a t t C$ ), which are recombined. Altogether, our results indicate that the RecA protein is a host factor specifically implicated in the cassette recruitment in the 
attIA site of the $V$. cholerae SCI during environmental stress conditions. Finally, RecA protein acts at several steps during integron recombination process (Figure 8). First, as said above, when antibiotic is used or when single-stranded cassette are released in cell by HGT, RecA favors the expression of the SOS-dependent integrase gene (Baharoglu and Mazel, 2011; Guerin et al., 2009). Second, since recA gene itself also belongs to the SOS regulon (Courcelle et al., 2001; Little et al., 1981), its expression is up-regulated (ten-fold within minutes (Courcelle et al., 2001; Renzette et al., 2005)). This release of RecA protein would allow RecA to be available to trigger its biological functions, upon which its stimulating effect on cassette recruitment at attIA SCI (Figure 8).

\section{Model of RecAvch mechanism of action}

RecA activates expression of a large number of genes during the SOS response (Krin et al., 2018). The mechanism of action of Rec $A_{V c h}$ on integron recombination could thus be the consequence of an indirect effect. Using a $V$. cholerae mutant strain lexAind- in which the SOS response is constitutively repressed, we showed that SOS response abrogation did not affect recombination rate and therefore that the observed Rec $_{V c h}$ effect on attIA $\times$ attC recombination does not involve any other protein belonging to the SOS regulon. Besides, we know that the E. coli RecA protein differs from the $V$. cholerae RecA protein in the extreme C-terminal part and that this part is known to modulate interactions with regulatory proteins (Figure S1) (Cox, 2007b). We decided to study the effect of the RecA $A_{E c}$ protein in attIA $\times$ attC recombination mediated by IntIA. We performed complementation of the V. cholerae ArecA mutant by the $r e c A_{E c}$ gene. We observed that the RecA $A_{E c}$ protein fully restores the recombination activity, meaning that both RecA proteins may insure cassette insertion in the attIA site.

Contrary to the attC $\times$ attIA reaction, we did not observe any influence of RecA $\mathrm{V}_{V c h}$ on $a t t C \times$ attC reaction mediated by IntIA in $V$. cholerae, while both reactions require ss attC site on double-stranded DNA molecule, we still observed an important effect of the RecA ${ }_{V c h}$ protein. Then, whatever the way to deliver $a t t C$ sites, i.e. single-stranded (conjugation and replicative assays) or double-stranded (replicative assay), we observed an important effect of the Rec $A_{V c h}$ protein in attIA $\times$ attC recombination. Altogether, these results show that observed Rec $A_{V c h}$ effect is not linked to the regulation of the attC sites folding. We therefore suppose that the attIA $\times$ attC synaptic complex formed during the recombination could impede the aHJ resolution by blocking replication fork progression. The effect of RecA would be to bind the 
ss attC sites and help to maintain the attC site in a non-folded single-stranded form destabilizing the synaptic complex and favoring the replicative resolution step. Note that we did not observe this $\operatorname{Rec}_{V c h}$ effect on $a t t C \times a t t C$ recombination suggesting a differential configuration between both $a t t C \times a t t C$ and $a t t I A \times a t t C$ complexes. Indeed, synapses formed during both reactions are known to be different since involving respectively two flexible bs attC sites and, a flexible bs attC and a stiffer ds attI (Demarre et al., 2007). Such differences would explain that the synapse architecture for the $a t t I A \times a t t C$ reaction versus $a t t C \times a t t C$ may potentially requires the assistance of additional accessory proteins. Furthermore, the attC $\times$ attC reaction can be catalyzed by IntIA in $E$. coli while, productive attIA $\times$ attC reactions are almost undetectable in this host (Biskri et al., 2005).

\section{Host factor recruitment differs between Mobile and Sedentary Chromosomal Integrons}

Biskri et al. previously observed the integrase of the SCI of $V$. cholerae recombines 2000 fold less efficiently during attIA $\times$ attC reaction, when expressed in a heterologous host such as E. coli (Biskri et al., 2005). This observation suggests that IntIA requires host factors that are absent or too divergent in E. coli to carry out this reaction (Biskri et al., 2005). Here, we confirmed this result and failed at recovering attIA $\times$ attC recombination by expressing Rec $_{V c h}$ in E. coli, suggesting that the Rec $A_{V c h}$ protein is not the missing factor in E. coli impeding this reaction to efficiently occur. This is not surprising since, even though they show some slight variations in the $C$ terminal part, $\operatorname{Rec}_{E c}$ and $\operatorname{Rec} \mathrm{A}_{V c h}$ present a very high level of identity (80\%, Figure S2). Besides, this is in accordance with the fact that Rec $\mathrm{A}_{E c}$ was able to functionally complement the absence of $\operatorname{Rec}_{V c h}$ to achieve this reaction in $V$. cholerae (Figure 3A). Therefore, we hypothesize that other specific $V$. cholerae host factors are missing in E. coli.

In contrast, RecA is not involved in any reactions mediated by IntI1 (neither in E. coli or V. cholerae) and all these reactions are efficient in V. cholerae. Therefore, while SCI cassette recombination activity seems restricted to a given host, MI recombination seems efficient in different species suggesting a host factor recruitment more stringent for SCIs compare to MIs. This is consistent with the observed widespread MIs dissemination among bacterial species. The evolutionary success of class 1 MIs clearly reflects the fact that they are functional in a wide range of bacterial hosts. This could result from a co-evolution between the integrase IntI1 and its attI1 site to allow that cassette recombination takes place in absence of specific accessory proteins. Both IntI and attI level of divergence, 45\% between IntIA and IntI1 (Demarre et al., 2007) and the lack of identity between attIA and attI1 sites, likely reflect their 
functioning differences. Indeed, attI1 site harbors supplementary IntI1 binding sites, the direct repeats (DRs, Figure S3), that constitute accessory sequences to which the integrase is able to bind (Gravel et al., 1998). These DRs favor attI1 × attC reaction (Partridge et al., 2000). However, such sequences are not common features to the majority of attI sites (Nield et al., 2001) and, for instance, the attIA site of the V. cholerae SCI does not harbor any DRs (Figure S3). Upon mobilization of integrons, attI sites could have evolved and acquired such motifs (e.g. DRs), to replace trans-acting host factors necessary for the attI1 $\times$ attC reactions. For instance, DRs of attI1 site acting as topological filters (Partridge et al., 2000) could therefore replace host factors that regulate supercoiling.

\section{Natural transformation represents an efficient pathway for SCI cassette recruitment}

HGT contributes to the emergence of pathogens and the spread of virulence factors, and also, enables many disease-causing bacteria to rapidly evolve in response to environmental pressures such as antibiotic use (Blokesch, 2016; de la Cruz and Davies, 2000; Frost et al., 2005; Gogarten et al., 2002; Ochman et al., 2005; Prudhomme et al., 2006; Waldor and Mekalanos, 1996). Among HGT processes, natural competence is able to mediate the absorption and exchange of free DNA when sufficient homology is present between the incoming DNA and the bacterial genome (Chen and Dubnau, 2004; Griffith, 1928). Here, we demonstrated that acquisition of material during natural transformation can be also mediated by homology independent DNA mechanism, notably by exchanging and recruiting gene cassettes inside integrons in an integrase dependent way. We used the causative agent of the diarrheal disease cholera, Vibrio cholerae, responsible for seven major pandemics since 1817, the latter of which is still ongoing (Lippi et al., 2016). Cassette recruitments occur directly in the attIA site of the SCI contained in V. cholerae where they are expressed and selected if procuring an advantage to the Vibrio cholerae strain. The natural bacterial competence has already been demonstrated for at least 80 species of bacteria but remains little explored and is probably underestimated (Johnsborg et al., 2007; Johnston et al., 2014). Notably, natural transformation is largely widespread among Vibrionaceae where SCIs are mainly found and constitute very plastic regions (Le Roux and Blokesch, 2018; Matthey et al., 2019). Here, we validated a new pathway for integron cassette recruitment in SCIs triggered by natural transformation probably involved in adaptation and making integrons important motors of evolution in Vibrionaceae species. 
678

679

680

681

682

683

684

685

686

687

688

689

690

691

692

693

694

695

696

697

698

699

700

701

702

703

704

705

706

707

708

709

710

711

\section{SUPPLEMENTARY DATA}

Table S1: Bacterial strains used in this study

Table S2: Plasmids used in this study

Table S3: Primers used in this study

Figure S1: Electrophoretic analysis of reactional substrates

Figure S2: Alignment of the Rec $A_{E c}$ and $\operatorname{Rec}_{V c h}$ protein sequences

Figure S3: attI site structures

\section{ACKNOWLEDGEMENT}

We would like to thank Evelyne Krin for providing the N16961 recA mutant. We also thank all the lab members for helpful discussion. We also thank Sandra Arroyo-Beck and Marcos Manero for their experimental help.

\section{FUNDING}

This work was supported by the French Government's Investissement d'Avenir program Laboratoire d'Excellence 'Integrative Biology of Emerging Infectious Diseases' [ANR-10LABX-62- IBEID] and 'Fondation pour la Recherche Médicale’.

\section{CONFLICT OF INTEREST}

None declared

\section{REFERENCES}

Baharoglu, Z., Bikard, D., and Mazel, D. (2010). Conjugative DNA transfer induces the bacterial SOS response and promotes antibiotic resistance development through integron activation. PLoS Genet 6, e1001165.

Baharoglu, Z., Krin, E., and Mazel, D. (2012). Connecting environment and genome plasticity in the characterization of transformation-induced SOS regulation and carbon catabolite control of the Vibrio cholerae integron integrase. J Bacteriol 194, 1659-1667.

Baharoglu, Z., and Mazel, D. (2011). Vibrio cholerae triggers SOS and mutagenesis in response to a wide range of antibiotics: a route towards multiresistance. Antimicrob Agents Chemother 55, 2438-2441.

Biskri, L., Bouvier, M., Guerout, A.M., Boisnard, S., and Mazel, D. (2005). Comparative Study of Class 1 Integron and Vibrio cholerae Superintegron Integrase Activities. J Bacteriol $187,1740-1750$. 
Biskri, L., and Mazel, D. (2003). Erythromycin esterase gene ere(A) is located in a functional gene cassette in an unusual class 2 integron. Antimicrob Agents Chemother 47, 3326-3331. Blokesch, M. (2016). Natural competence for transformation. Curr Biol 26, 3255. Bouvier, M., Demarre, G., and Mazel, D. (2005). Integron cassette insertion: a recombination process involving a folded single strand substrate. Embo J 24, 4356-4367. features of single-stranded integron cassette attC sites and their role in strand selection. PLoS Genet 5, e1000632. Cambray, G., Guerout, A.M., and Mazel, D. (2010). Integrons. Annu Rev Genet 44, 141-166. Cambray, G., Sanchez-Alberola, N., Campoy, S., Guerin, E., Da Re, S., Gonzalez-Zorn, B., Ploy, M.C., Barbe, J., Mazel, D., and Erill, I. (2011). Prevalence of SOS-mediated control of integron integrase expression as an adaptive trait of chromosomal and mobile integrons. Mobile DNA 2, 6.

Chen, I., and Dubnau, D. (2004). DNA uptake during bacterial transformation. Nat Rev Microbiol 2, 241-249.

Cherepanov, P.P., and Wackernagel, W. (1995). Gene disruption in Escherichia coli: TcR and KmR cassettes with the option of Flp-catalyzed excision of the antibiotic-resistance determinant. Gene 158, 9-14. integrases preferentially recognize the adjacent cognate attI site in recombination with a 59-be site. Mol Microbiol 46, 1415-1427.

Courcelle, J., Khodursky, A., Peter, B., Brown, P.O., and Hanawalt, P.C. (2001). Comparative gene expression profiles following UV exposure in wild-type and SOS-deficient Escherichia coli. Genetics 158, 41-64.

Cox, M.M. (2007a). Motoring along with the bacterial RecA protein. Nat Rev Mol Cell Biol 8, 127-138.

Cox, M.M. (2007b). Regulation of bacterial RecA protein function. Crit Rev Biochem Mol Biol 42, 41-63.

740 Cronan, J.E. (2003). Cosmid-based system for transient expression and absolute off-to-on transcriptional control of Escherichia coli genes. J Bacteriol 185, 6522-6529. 
da Fonseca, E.L., and Vicente, A.C. (2012). Functional characterization of a Cassette-specific promoter in the class 1 integron-associated qnrVC1 gene. Antimicrob Agents Chemother 56, 3392-3394.

de la Cruz, F., and Davies, J. (2000). Horizontal gene transfer and the origin of species: lessons from bacteria. Trends in Microbiology 8, 128-133.

de Lemos Martins, F., Fournes, F., Mazzuoli, M.V., Mazel, D., and Val, M.E. (2018). Vibrio cholerae chromosome 2 copy number is controlled by the methylation-independent binding of its monomeric initiator to the chromosome 1 crtS site. Nucleic Acids Res 46, 10145-10156.

Demarre, G., Frumerie, C., Gopaul, D.N., and Mazel, D. (2007). Identification of key structural determinants of the IntI1 integron integrase that influence attC $x$ attI1 recombination efficiency. Nucleic Acids Res 35, 6475-6489.

Domingues, S., Harms, K., Fricke, W.F., Johnsen, P.J., da Silva, G.J., and Nielsen, K.M. (2012). Natural transformation facilitates transfer of transposons, integrons and gene cassettes between bacterial species. PLoS Pathog 8, e1002837.

Escudero, J.A., Loot, C., Nivina, A., and Mazel, D. (2015). The Integron: Adaptation On Demand. Microbiology spectrum 3, MDNA3-0019-2014.

Francia, M.V., Zabala, J.C., de la Cruz, F., and Garcia-Lobo, J.M. (1999). The IntI1 integron integrase preferentially binds single-stranded DNA of the attC site. Journal of Bacteriology 181, 6844-6849.

Frost, L.S., Leplae, R., Summers, A.O., and Toussaint, A. (2005). Mobile genetic elements: the agents of open source evolution. Nat Rev Microbiol 3, 722-732.

Gogarten, J.P., Doolittle, W.F., and Lawrence, J.G. (2002). Prokaryotic evolution in light of gene transfer. Mol Biol Evol 19, 2226-2238.

Goldberg, I., and Mekalanos, J.J. (1986). Effect of a recA mutation on cholera toxin gene amplification and deletion events. J Bacteriol 165, 723-731.

Gravel, A., Fournier, B., and Roy, P.H. (1998). DNA complexes obtained with the integron integrase IntI1 at the attI1 site. Nucleic Acids Research 26, 4347-4355.

Griffith, F. (1928). The Significance of Pneumococcal Types. J Hyg (Lond) 27, 113-159.

Guerin, E., Cambray, G., Sanchez-Alberola, N., Campoy, S., Erill, I., Da Re, S., GonzalezZorn, B., Barbe, J., Ploy, M.C., and Mazel, D. (2009). The SOS response controls integron recombination. Science 324, 1034.

Jacquier, H., Zaoui, C., Sanson-le Pors, M.J., Mazel, D., and Bercot, B. (2009). Translation regulation of integrons gene cassette expression by the attC sites. Mol Microbiol 72, 14751486. 
Johansson, C., Kamali-Moghaddam, M., and Sundstrom, L. (2004). Integron integrase binds to bulged hairpin DNA. Nucleic Acids Res 32, 4033-4043.

Johnsborg, O., Eldholm, V., and Havarstein, L.S. (2007). Natural genetic transformation: prevalence, mechanisms and function. Res Microbiol 158, 767-778.

Johnston, C., Campo, N., Berge, M.J., Polard, P., and Claverys, J.P. (2014). Streptococcus pneumoniae, le transformiste. Trends Microbiol 22, 113-119.

Kowalczykowski, S.C. (2000). Initiation of genetic recombination and recombinationdependent replication. Trends Biochem Sci 25, 156-165.

Krin, E., Cambray, G., and Mazel, D. (2014). The superintegron integrase and the cassette promoters are co-regulated in Vibrio cholerae. PLoS One 9, e91194.

Krin, E., Pierle, S.A., Sismeiro, O., Jagla, B., Dillies, M.A., Varet, H., Irazoki, O., Campoy, S., Rouy, Z., Cruveiller, S., et al. (2018). Expansion of the SOS regulon of Vibrio cholerae through extensive transcriptome analysis and experimental validation. BMC Genomics 19, 373.

Le Roux, F., Binesse, J., Saulnier, D., and Mazel, D. (2007). Construction of a Vibrio splendidus mutant lacking the metalloprotease gene vsm by use of a novel counterselectable suicide vector. Appl Environ Microbiol 73, 777-784.

Le Roux, F., and Blokesch, M. (2018). Eco-evolutionary Dynamics Linked to Horizontal Gene Transfer in Vibrios. Annu Rev Microbiol 72, 89-110.

Lippi, D., Gotuzzo, E., and Caini, S. (2016). Cholera. Microbiology spectrum 4. Little, J.W., Mount, D.W., and Yanisch-Perron, C.R. (1981). Purified lexA protein is a repressor of the recA and lexA genes. Proc Natl Acad Sci U S A 78, 4199-4203.

Loot, C., Bikard, D., Rachlin, A., and Mazel, D. (2010). Cellular pathways controlling integron cassette site folding. EMBO J 29, 2623-2634.

Loot, C., Ducos-Galand, M., Escudero, J.A., Bouvier, M., and Mazel, D. (2012). Replicative resolution of integron cassette insertion. Nucleic Acids Res 40, 8361-8370.

Loot, C., Nivina, A., Cury, J., Escudero, J.A., Ducos-Galand, M., Bikard, D., Rocha, E.P., and Mazel, D. (2017). Differences in Integron Cassette Excision Dynamics Shape a Trade-Off between Evolvability and Genetic Capacitance. mBio 8.

Loot, C., Parissi, V., Escudero, J.A., Amarir-Bouhram, J., Bikard, D., and Mazel, D. (2014). The integron integrase efficiently prevents the melting effect of Escherichia coli singlestranded DNA-binding protein on folded attC sites. J Bacteriol 196, 762-771.

Lusetti, S.L., and Cox, M.M. (2002). The bacterial RecA protein and the recombinational DNA repair of stalled replication forks. Annual review of biochemistry 71, 71-100. 

DinI protein stabilizes RecA protein filaments. J Biol Chem 279, 30037-30046. Marvig, R.L., and Blokesch, M. (2010). Natural transformation of Vibrio cholerae as a tool-optimizing the procedure. BMC Microbiol 10, 155. Neighbor predation linked to natural competence fosters the transfer of large genomic regions in Vibrio cholerae. Elife 8.

Mazel, D., Dychinco, B., Webb, V.A., and Davies, J. (1998). A distinctive class of integron in the Vibrio cholerae genome. Science 280, 605-608. Meibom, K.L., Blokesch, M., Dolganov, N.A., Wu, C.Y., and Schoolnik, G.K. (2005). Chitin induces natural competence in Vibrio cholerae. Science 310, 1824-1827. Nield, B.S., Holmes, A.J., Gillings, M.R., Recchia, G.D., Mabbutt, B.C., Nevalainen, K.M., and Stokes, H.W. (2001). Recovery of new integron classes from environmental DNA. FEMS Microbiol Lett 195, 59-65.

827 Nivina, A., Escudero, J.A., Vit, C., Mazel, D., and Loot, C. (2016). Efficiency of integron cassette insertion in correct orientation is ensured by the interplay of the three unpaired features of attC recombination sites. Nucleic Acids Res 44, 7792-7803. of gene transfer and exchange. Proc Natl Acad Sci U S A 102 Suppl 1, 6595-6599. Associated with Antimicrobial Resistance. Clin Microbiol Rev 31.

834 Partridge, S.R., Recchia, G.D., Scaramuzzi, C., Collis, C.M., Stokes, H.W., and Hall, R.M. (2000). Definition of the attI1 site of class 1 integrons [In Process Citation]. Microbiology 146, 2855-2864.

Prudhomme, M., Attaiech, L., Sanchez, G., Martin, B., and Claverys, J.P. (2006). Antibiotic stress induces genetic transformability in the human pathogen Streptococcus pneumoniae. Science 313, 89-92.

840 Renzette, N., Gumlaw, N., Nordman, J.T., Krieger, M., Yeh, S.P., Long, E., Centore, R., 841 Boonsombat, R., and Sandler, S.J. (2005). Localization of RecA in Escherichia coli K-12 using RecA-GFP. Mol Microbiol 57, 1074-1085.

843 Rowe-Magnus, D.A., Guerout, A.M., and Mazel, D. (2002). Bacterial resistance evolution by recruitment of super-integron gene cassettes. Mol Microbiol 43, 1657-1669. 
845 Rowe-Magnus, D.A., Guerout, A.M., Ploncard, P., Dychinco, B., Davies, J., and Mazel, D. (2001). The evolutionary history of chromosomal super-integrons provides an ancestry for multiresistant integrons. Proc Natl Acad Sci U S A 98, 652-657.

848 Shinohara, A., and Ogawa, T. (1999). Rad51/RecA protein families and the associated proteins in eukaryotes. Mutat Res 435, 13-21.

850 Stokes, H.W., and Hall, R.M. (1989). A novel family of potentially mobile DNA elements encoding site-specific gene-integration functions: integrons. Molecular Microbiology 3, 16691683.

Stokes, H.W., and Hall, R.M. (1991). Sequence analysis of the inducible chloramphenicol resistance determinant in the Tn1696 integron suggests regulation by translational attenuation. Plasmid 26, 10-19. Antibiotic Resistance Dissemination by Regulating Integron Integrase Expression in Biofilms. mBio 7.

Szekeres, S., Dauti, M., Wilde, C., Mazel, D., and Rowe-Magnus, D.A. (2007). Chromosomal toxin-antitoxin loci can diminish large-scale genome reductions in the absence of selection. Mol Microbiol 63, 1588-1605. engineering in Vibrio cholerae: a feasible approach to address biological issues. PLoS Genet 8, e1002472.

Waldor, M.K., and Mekalanos, J.J. (1996). Lysogenic conversion by a filamentous phage encoding cholera toxin. Science 272, 1910-1914.

\section{FIGURES LEGENDS}

\section{Figure 1: The integron.}

871 (A) The integron system in Vibrio cholerae.

$872 V$. cholerae sedentary chromosomal integron is located on second chromosome close to the termination site, Ter2. The four components of integron stable platform are shown: the integrase expressing gene, intIA, the two promoters, $\mathrm{P}_{\mathrm{C}}$ and $\mathrm{P}_{\text {int }}$ and the attIA recombination site (red triangle). The variable cassette array contains a large number of cassettes, which are represented by small arrows. Their expression level is reflected by the colour intensity of each arrow. Only the first cassettes of the array are expressed, and the subsequent ones can be seen as a low-cost cassette reservoir. Upon expression of the integrase (grey forms) cassette 
shuffling can occur through cassette excision ( $a t t C$ x attC) and integration in the first position in the array (attIA x attC).

(B) Integron cassette insertion in an attI site.

Recombination between the double-stranded attI site (bold red lines) and a single-stranded bottom attC site (green lines) ending a cassette is shown. Since we do not exactly know the nature of the cassettes (ss or ds), the top strand of the attC site is represented as a dotted line. The synaptic complex comprises both att sites bound by four integrase monomers (grey ovals). One strand from each att site is cleaved and transferred to form an atypical Holliday junction (aHJ). aHJ resolution implies a replication step. The origin of replication is represented by a grey circle and the newly synthesized leading and lagging strands by dashed lines. Both products are represented: the initial substrate resulting from the top strand replication, and the reactional product containing the inserted cassette and resulting from the bottom strand replication.

Figure 2: Cassette recruitment in V. cholerae SCI during horizontal gene transfer.

(A) Experimental setup of the cassette insertion assay

The pSW23T::attCaadA7 suicide vector is delivered to N16961 V. cholerae recipient strains containing an integrase expressing vector or the sole endogenous integrase, and the SCI. The delivering occurs by two horizontal gene transfer processes: conjugation from the $\beta 2163$ donor or natural transformation. As the pSW23T cannot replicate in $V$. cholerae recipient strains, recombinant clones can be selected on appropriate $\mathrm{Cm}$ containing plates to evaluate the recombination frequency (see also Results and Material and methods). The attCaadA7 site carried by the suicide vector is represented by a green triangle and the attIA site on the $V$. cholerae SCI by a red triangle.

(B) Frequency of insertion of the pSW23T::att $C_{a a d A 7}$ suicide vector into the attIA site. The recombination frequencies were calculated in N16961 V. cholerae wt, AintIA and lexAindstrains. Results correspond to recombination frequencies that were normalized after analysis of PCR reactions (Material and Methods). +IntIA: recipient strains transformed with the pBAD43 integrase expressing vector; -IntIA: control strains transformed with the empty pBAD43 vector. * correspond to the limits of detection. Values represent the mean of at least three independent experiments and error bars correspond to average deviations from the mean. 
(A) Experimental setup and frequency of insertion of the pSW23T::attCaadA7 suicide vector into the chromosomic attIA site. N16961 recipient strains transformed with the pBAD43 IntIA expressing vector were used (left panel). The recombination rates were calculated in N16961 V. cholerae wt and in the corresponding recA mutant (ArecA) and ectopic complemented ( $\Delta r e c A-a t t_{t n 7}:: \mathrm{P}_{\mathrm{LAC}}-r e c A_{V c h}$ and $\left.\Delta r e c A-a t t_{t n 7}:: \mathrm{P}_{\mathrm{LAC}}-r e c A_{E c}\right)$ strains (right panel).

(B) Experimental setup and frequency of insertion of the pSW23T::att $C_{a a d A 7}$ suicide vector into the attIA site located on plasmid. N16961 recipient strains transformed with both pBAD43 IntIA expressing vector and pSU384::attIA vector were used (left panel). The recombination rates were calculated in N16961 $\mathrm{V}$. cholerae wt and in the corresponding recA mutant strains ( $\triangle r e c A$, right panel).

For both (A) and (B), results correspond to recombination frequencies that were normalized after analysis of PCR reactions (Material and Methods). +IntIA: recipient strains transformed with the pBAD43 integrase expressing vector; -IntIA: control strains transformed with the empty pBAD43 vector. * correspond to the limits of detection. Values represent the mean of at least three independent experiments and error bars correspond to average deviations from the mean.

Figure 4: Effect of the RecA protein on attC $\times$ attC recombination in $V$. cholerae.

(A) Experimental setup and frequency of insertion of the pSW23T::attCaadA7 suicide vector into VCR sites of the SCI. N16961 recipient strains deleted for the attIA site ( $\triangle a t t I A$ strains) and transformed with the pBAD43 IntIA expressing vector were used (left panel). The recombination rates were calculated in N16961 V. cholerae $\Delta a t t I A$ and in the corresponding recA mutant strain ( $\triangle a t t I A \Delta r e c A$, right panel).

(B) Experimental setup and frequency of insertion of the pSW23T::attCaadA7 suicide vector into the $a t t C_{a a d A 1}$ site located on the pBAD43 plasmid. N16961 recipient strains deleted for the attIA site ( $\triangle a t t I A$ strains) and transformed with the IntIA expressing and attCaadA1 containing pBAD43 vector were used (left panel). The recombination rates were calculated in N16961 V. cholerae $\triangle a t t I A$ and the corresponding recA mutant strain ( $\triangle a t t I A \Delta r e c A$, right panel). after analysis of PCR reactions and sequencing (Material and Methods). +IntIA: recipient strains transformed with the pBAD43 integrase expressing vector; -IntIA: control strains transformed with the empty pBAD43 vector. * correspond to the limits of detection. Values 
represent the mean of at least three independent experiments and error bars correspond to average deviations from the mean.

Figure 5: Effect of the RecA protein on attI1 $\times$ attC recombination mediated by IntI1 in V. cholerae.

(A) Experimental setup and frequency of insertion of the pSW23T::attCaadA7 suicide vector into the attI1 site located in the SCI platform. N16961 recipient strains with an attI1 site in place of the attIA site ( $\triangle a t t I A:: a t t I 1$ strains) were transformed with the pBAD43 IntI1 expressing vector (left panel). The recombination rates were calculated in N16961 V. cholerae

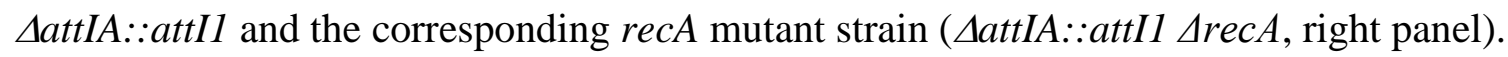

(B) Experimental setup and frequency of insertion of the pSW23T::attC aadA7 suicide vector into the attI1 site located on a plasmid. N16961 recipient strain deleted for the attIA site (AattIA::attI1 strain) and transformed with both pBAD43 IntI1 expressing vector and pSU38 $4:$ attI1 vector were used (left panel). The recombination rates were calculated in $V$. cholerae $\Delta a t t I A:: a t t I 1$ and the corresponding recA mutant strain ( $\triangle a t t I A:: a t t I 1 \Delta r e c A$, right panel).

For both (A) and (B), results correspond to recombination frequencies that were normalized after analysis of PCR reactions (Material and Methods). +IntIA: recipient strains transformed with the pBAD43 integrase expressing vector; -IntIA: control strains transformed with the empty pBAD43 vector. * correspond to the limits of detection. Values represent the mean of at least three independent experiments and error bars correspond to average deviations from the mean.

Figure 6: Effect of the RecA protein on recombination reactions catalysed by IntI1 and IntIA in E. coli.

(A) Experimental setup and frequency of insertion of the pSW23T::att $C_{a a d A 7}$ suicide vector into the att sites (attI1 or attIA) located on a plasmid. MG1655 recipient strains transformed with both pBAD43 IntI expressing vector (IntI1 or IntIA) and the pSU384::attI vector (attI1 or attIA) were used (left panel). The recombination rates were calculated in MG1655 and in the corresponding recA mutant $(\Delta r e c A)$ and ectopic complemented ( $\Delta r e c A-a t t_{t n}:: \mathrm{P}_{\mathrm{LAC}}-$ rec $\left.A_{V c h}\right)$ strains (right panels).

(B) Experimental setup and frequency of insertion of the pSW23T::attC $C_{a a d A 7}$ suicide vector into the VCR ${ }_{\text {Vca0441 }}$ site located on a plasmid. MG1655 recipient strains transformed with both 
pBAD43 IntI expressing vector (IntI1 or IntIA) and the pSU384::VCRvcA0441 vector were used (left panel). The recombination rates were calculated in MG1655 and in the corresponding

981

982

983

984

985

986

987

988

989

990

991

992

993

994

995

996

997

998

999

1000

1001

1002

1003

1004

1005

1006

1007

1008

1009

1010

1011 recA mutant strain ( $\triangle r e c A$, right panels).

For both (A) and (B), results correspond to recombination frequencies that were normalized after analysis of PCR reactions (Material and Methods). +IntI: recipient strains transformed with the pBAD43 integrase expressing vector; -IntI: control strains transformed with the empty pBAD43 vector. * correspond to the limits of detection. Values represent the mean of at least three independent experiments and error bars correspond to average deviations from the mean.

Figure 7: Effect of the RecA protein on attC $\times$ attI reaction when attC sites are carried by a replicative vector in $V$. cholerae.

(A) Experimental setup of the replicative assay. V. cholerae N16961 strains transformed with both pBAD18 IntIA expressing vector and the unidirectional-replicative vector, pTSC29::attC $C_{a a d A 7}$ were used. attCaadA7 sites (green triangles) were cloned in both orientations (double arrow) in the pTSC29 vector. Since pTSC29 has a thermosensitive origin of replication, recombination reaction is performed at $30^{\circ} \mathrm{C}$ and, to evaluate the recombination frequency, recombinant clones selection was performed on $\mathrm{Cm}$ containing plates at $42^{\circ} \mathrm{C}$ (see also Results and Material and methods). The attIA site on the $V$. cholerae SCI is represented by a red triangle.

(B) Folding of att $C_{a a d A 7}$ site on replicative vector. Replicated and template strands are coloured in red and grey respectively and grey circle represent IntIA monomers. bs: bottom strand; ts: top strand; Lag st temp: Lagging strand template; Lead st temp: Leading strand template.

(C) Frequency of insertion of the pTSC29::attCaadA7 unidirectional-replicative vector into the attIA site. The recombination rates were calculated in N16961 V. cholerae wt and in the corresponding recA mutant strains $(\triangle r e c A)$. Under plots, the orientation of $a t t C_{a a d A 7}$ bs on lagging or leading strand template (lag st temp or lead st temp) are indicated. Results correspond to recombination frequencies that were normalized after analysis of PCR reactions (Material and Methods). +IntI: recipient strains transformed with the pBAD18 integrase expressing vector; -IntI: control strains transformed with the empty pBAD18 vector. * correspond to the limits of detection. Values represent the mean of at least three independent experiments and error bars correspond to average deviations from the mean. 
1012 Figure 8: Snap shot of SCI recombination during horizontal gene transfer in Vibrio 1013 cholerae

1014 The SCI activity is represented and its connections with bacterial physiology. The steps, 1015 which involve the RecA protein, are indicated in green. HGT: horizontal gene transfer; ss: 1016 single-stranded; VCR: Vibrio cholerae Repeat sequences; SCI: sedentary chromosomal 1017 integron.

1018

1019

1020

1021

1022

1023

1024 
bioRxiv preprint doi: https://doi.org/10.1101/2020.11.24.395434; this version posted November 24, 2020. The copyright holder for this preprint (which was not certified by peer review) is the author/funder. All rights reserved. No reuse allowed without permission. 
A

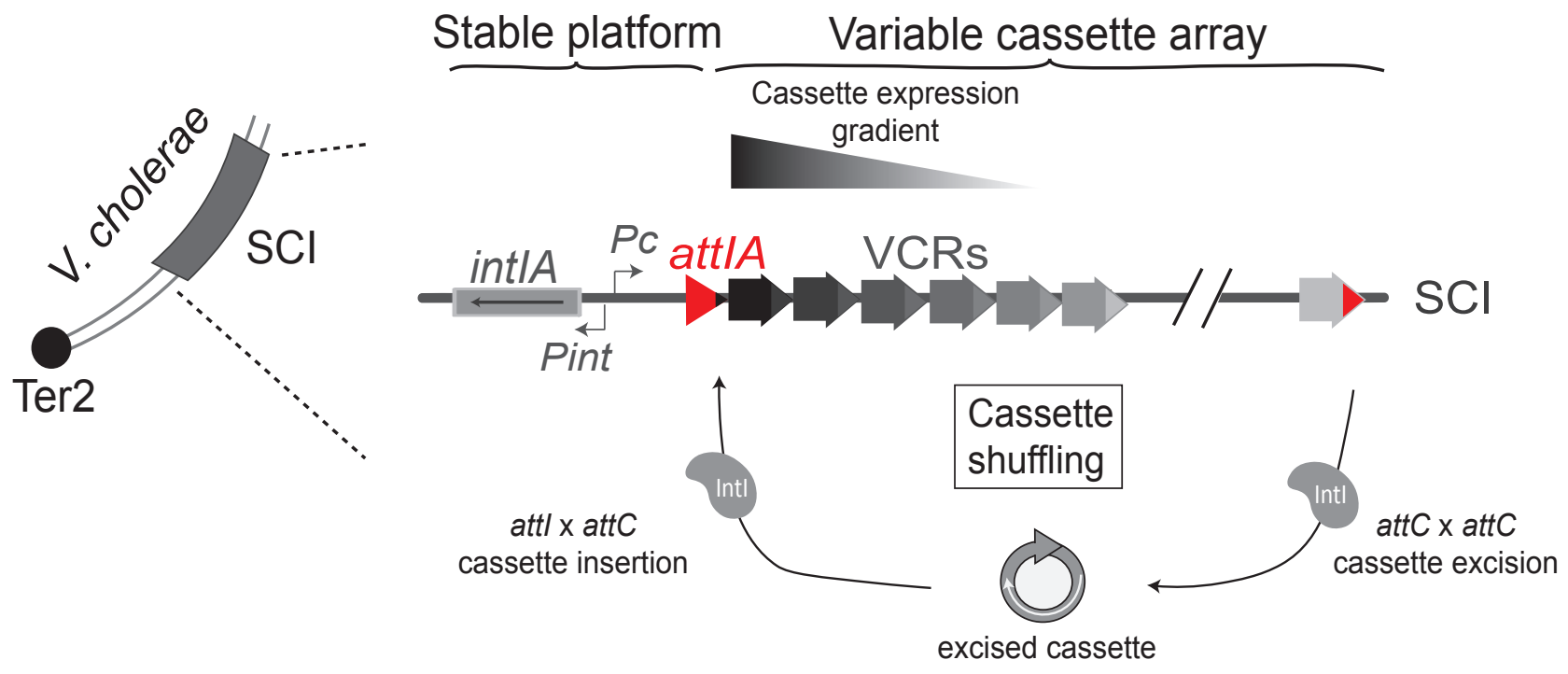

B

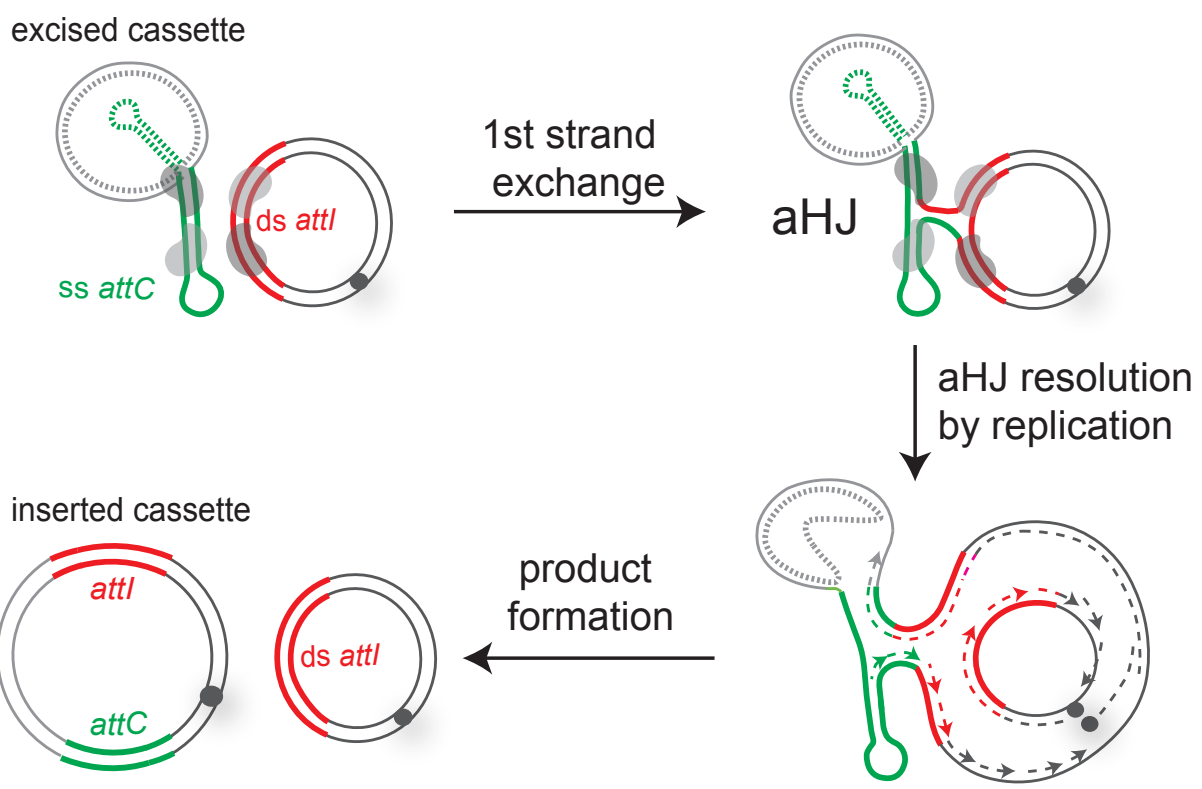

Figure 1 
A
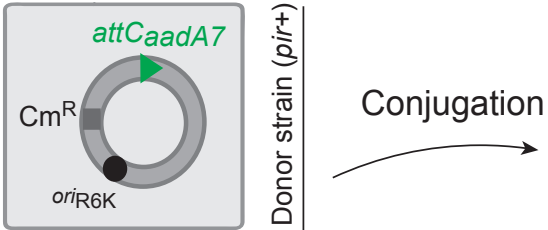

\section{Recipient strain (pir-) $\quad$ V. cholerae N16961}
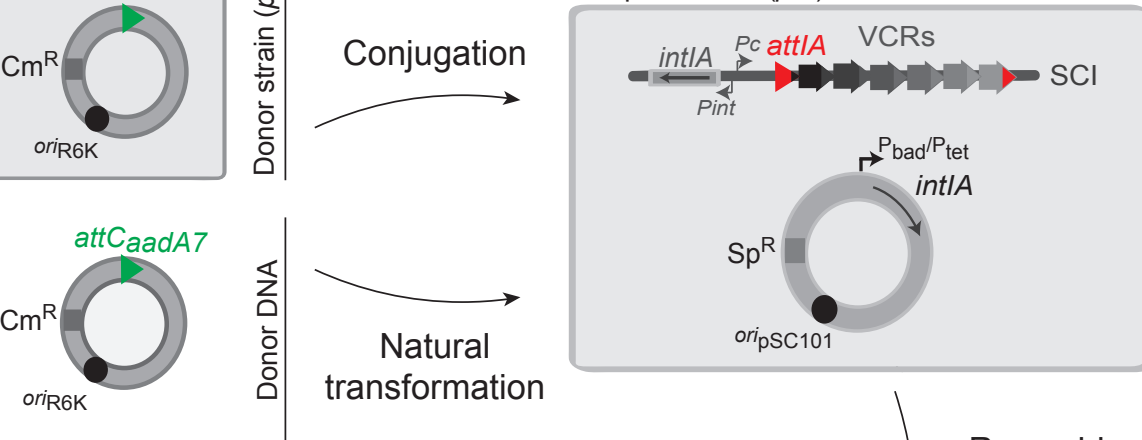

Recombination

$\mathrm{Cm}$ selection

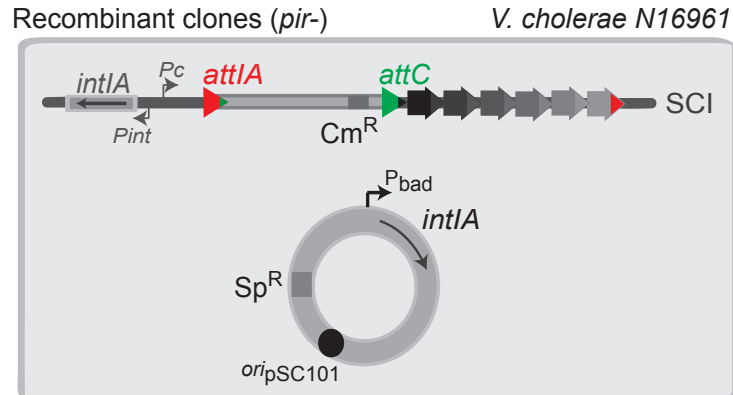

B
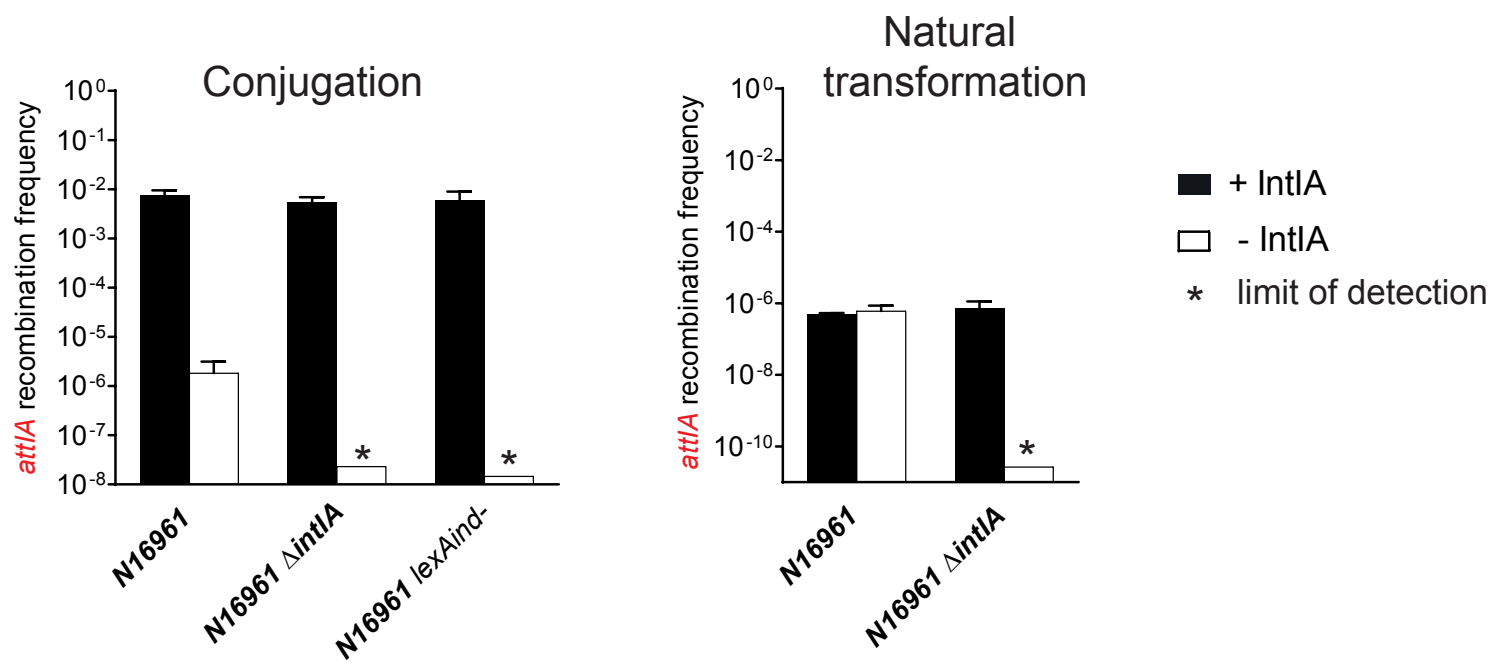
A

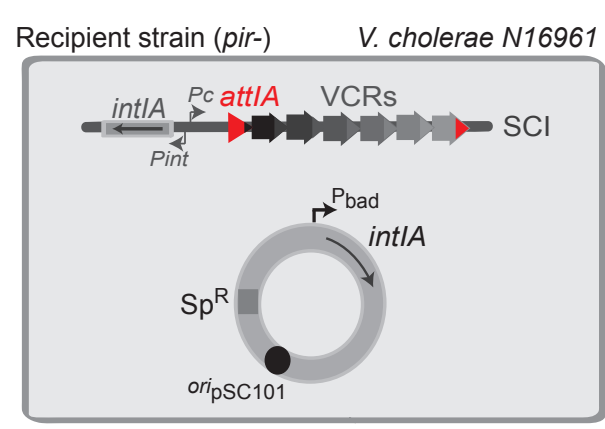

B

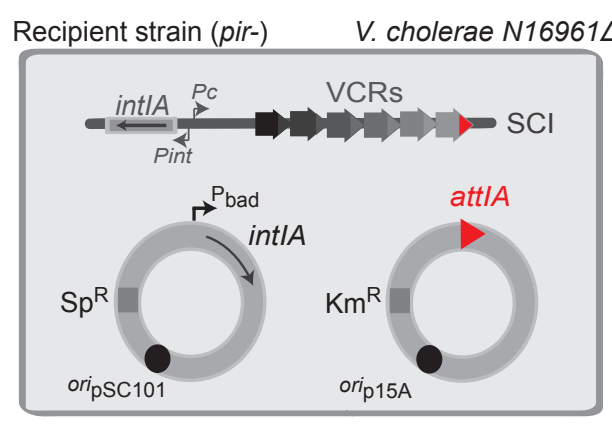

- $+\operatorname{Int} \mid \mathrm{A}$

$\square-\ln t \mid A$

* limit of detection
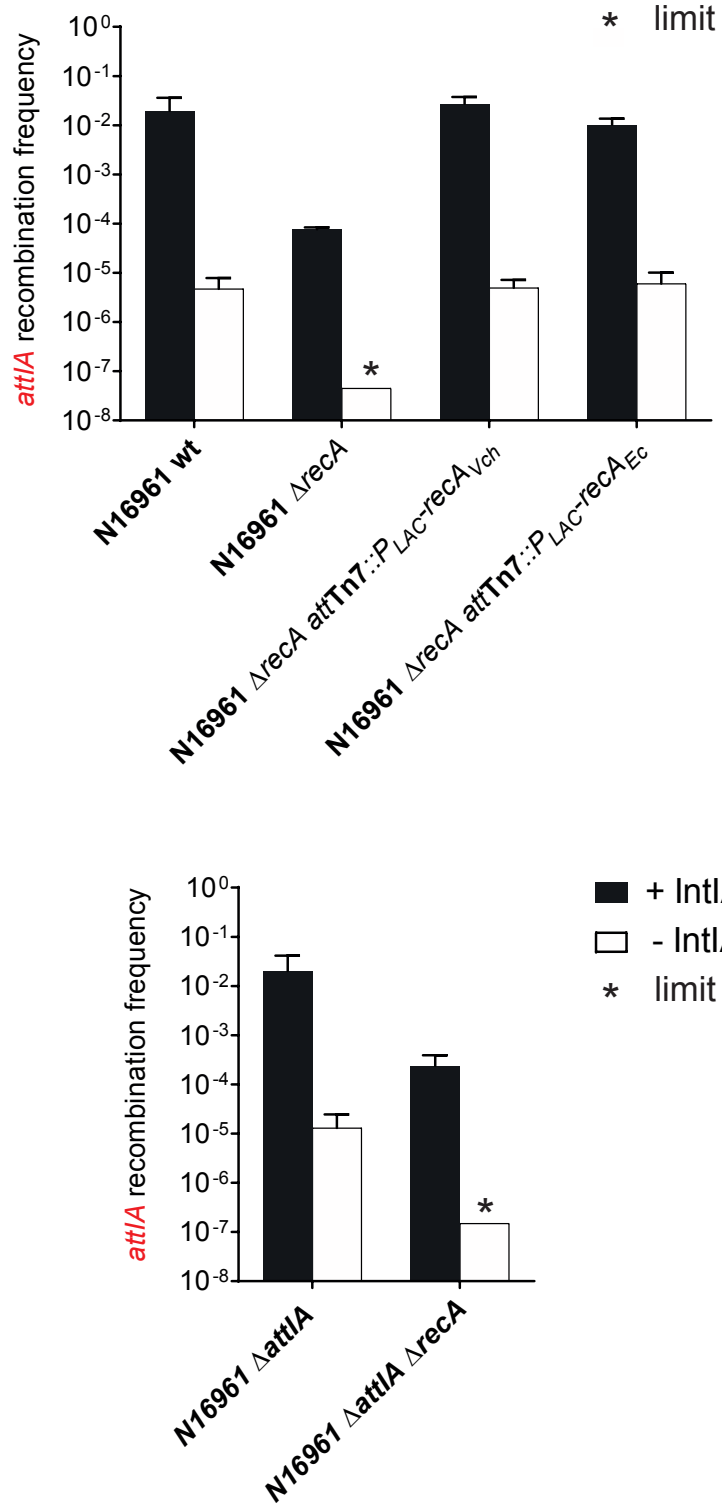
A

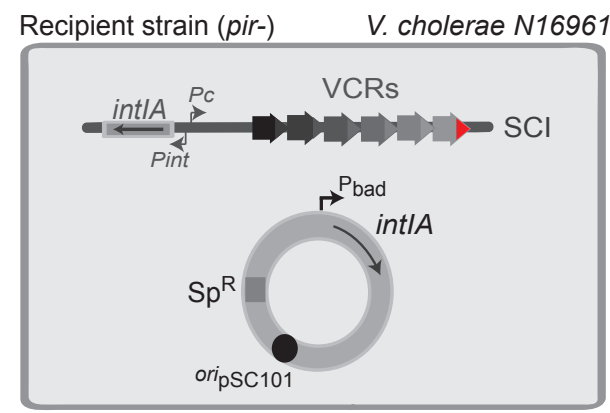

B

Recipient strain (pir-) V. cholerae N16961 $\quad$ attIA

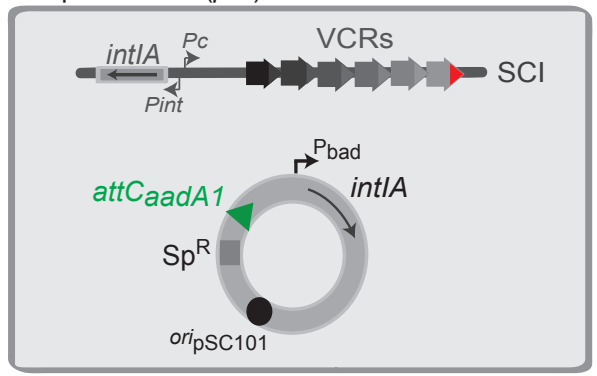

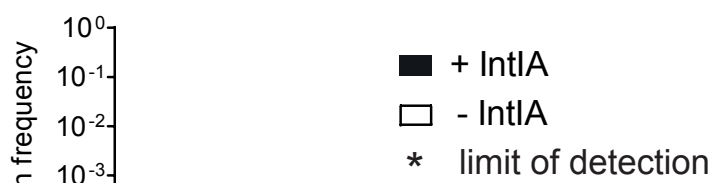
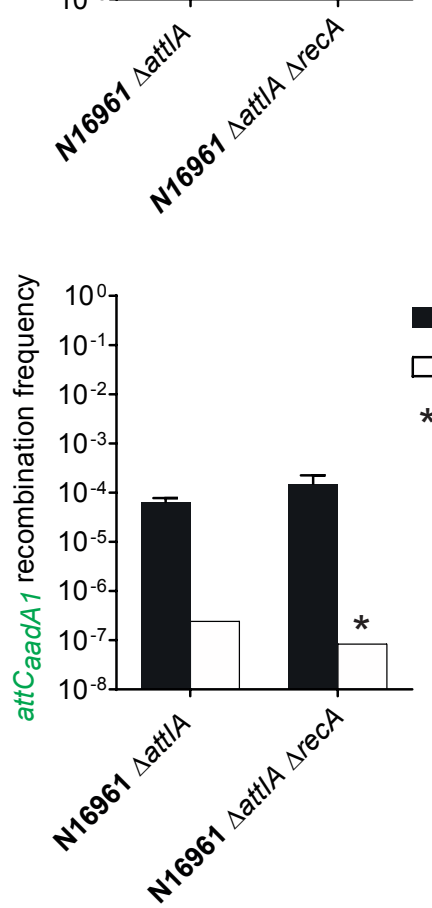

Figure 4 

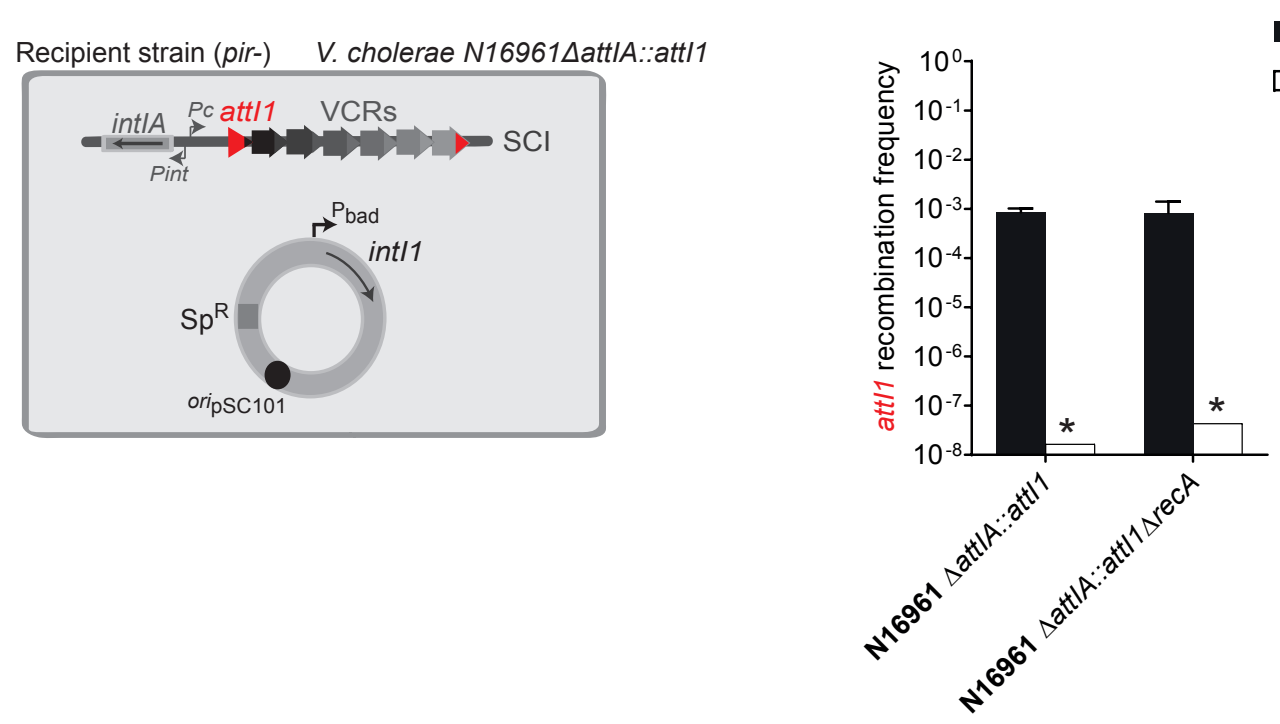

B
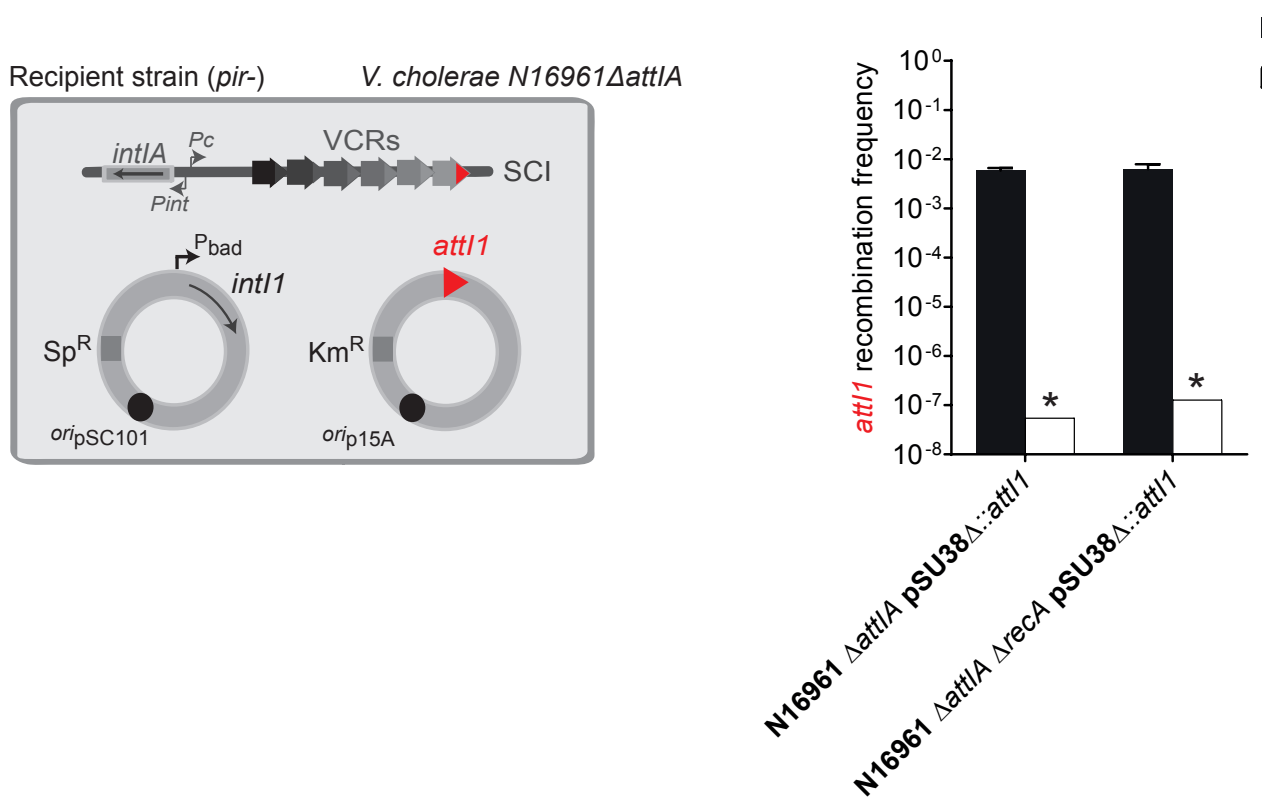

\section{Figure 5}


A

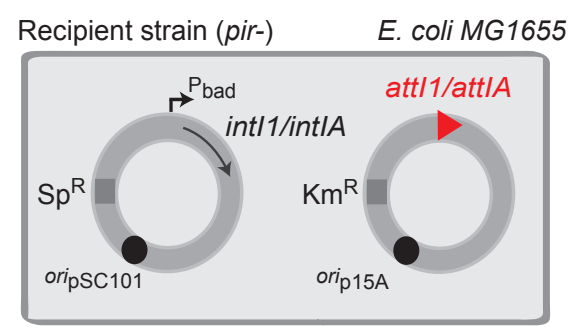

B

Recipient strain (pir-)

E. coli MG1655

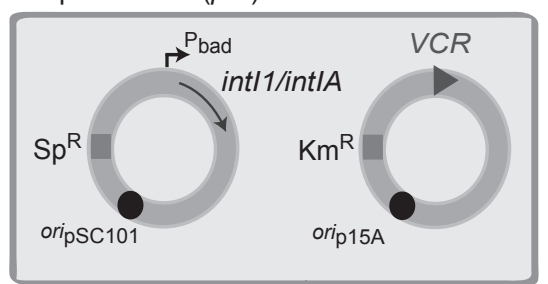

int/1

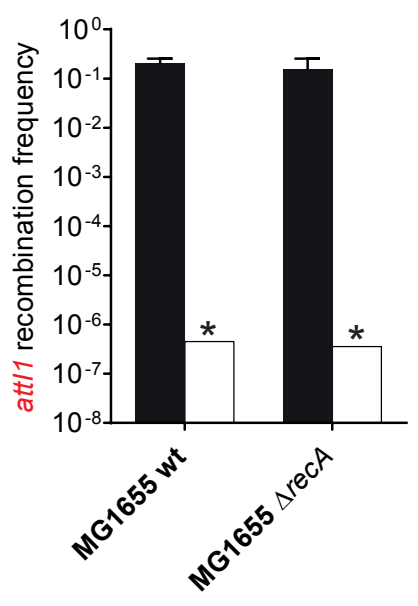

int/1

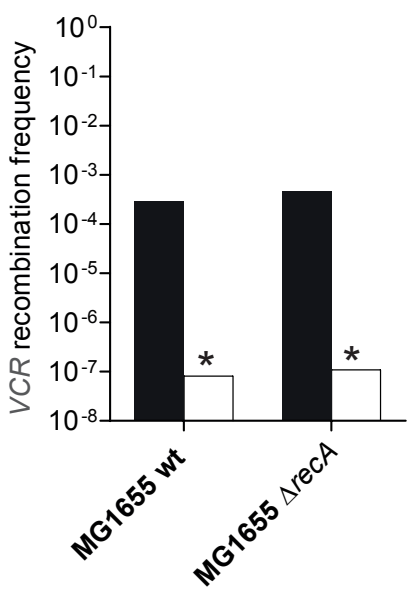

intIA

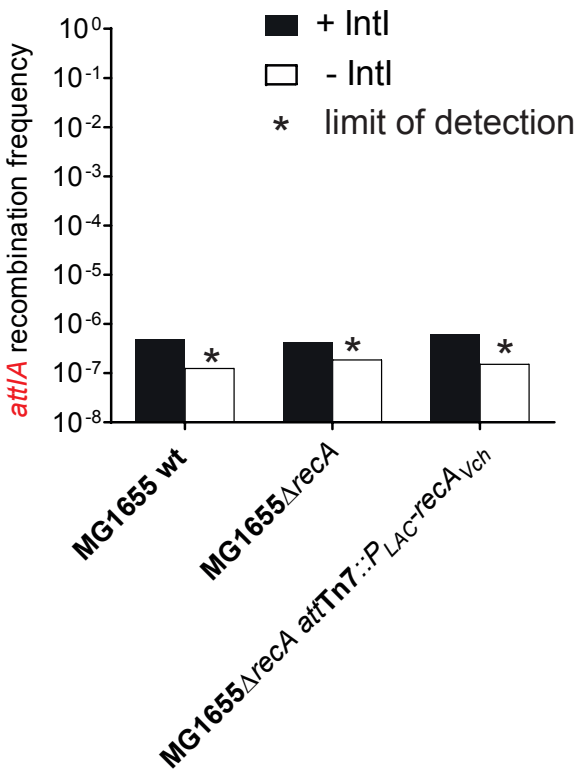

intIA

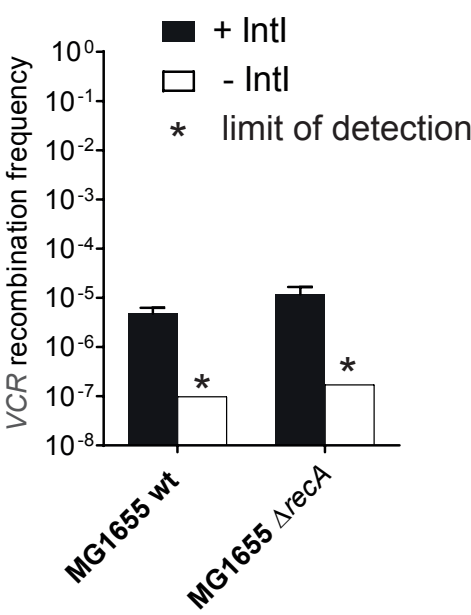

\section{Figure 6}




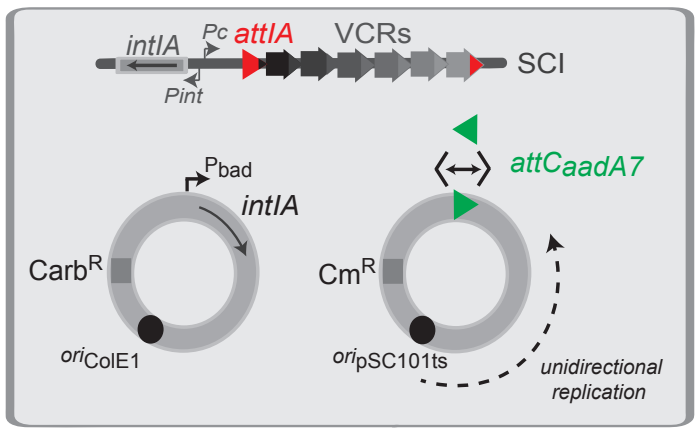

attCbs carried on the lagging strand template

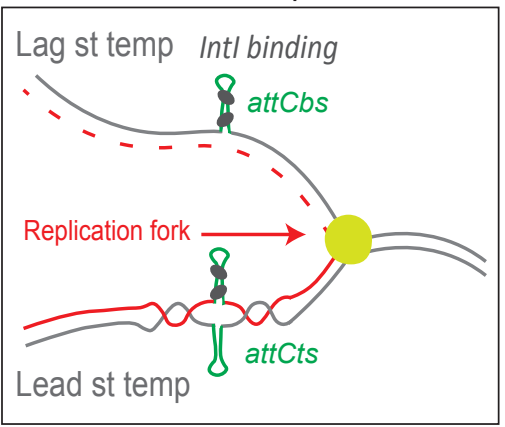

Recombination at $30^{\circ} \mathrm{C}$ $\mathrm{Cm}$ selection at $42^{\circ} \mathrm{C}$

attCbs carried on the leading

Recombinant clones

V. cholerae N16961
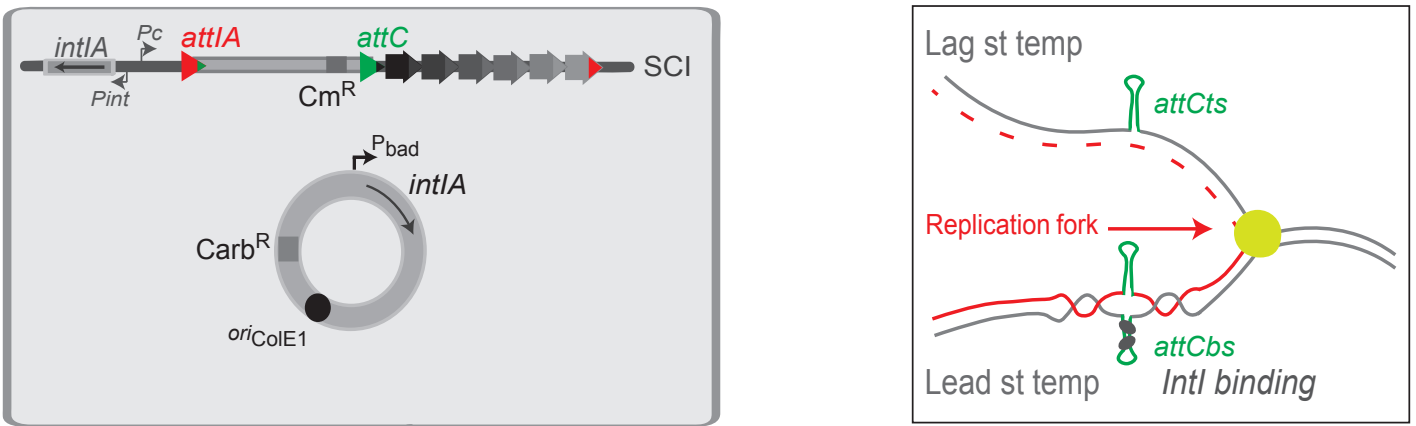

C

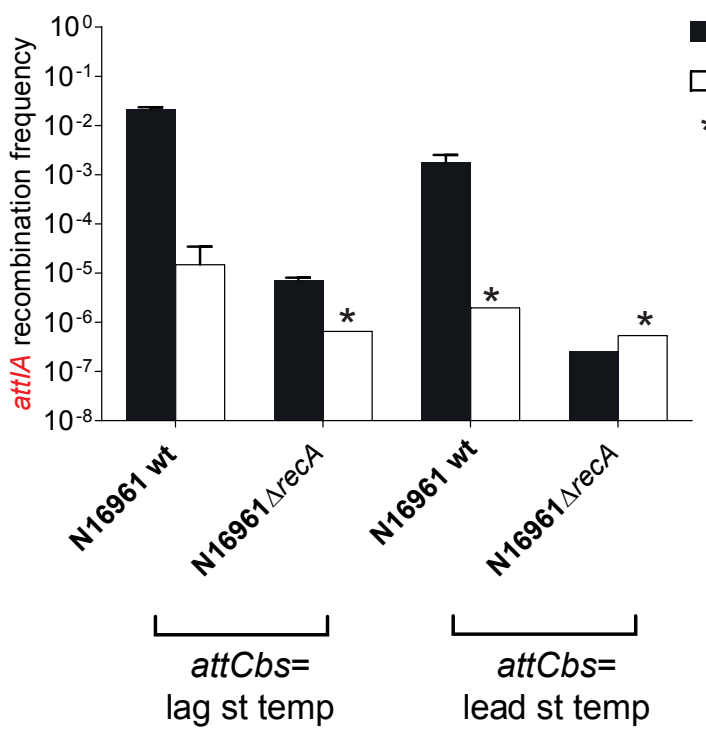




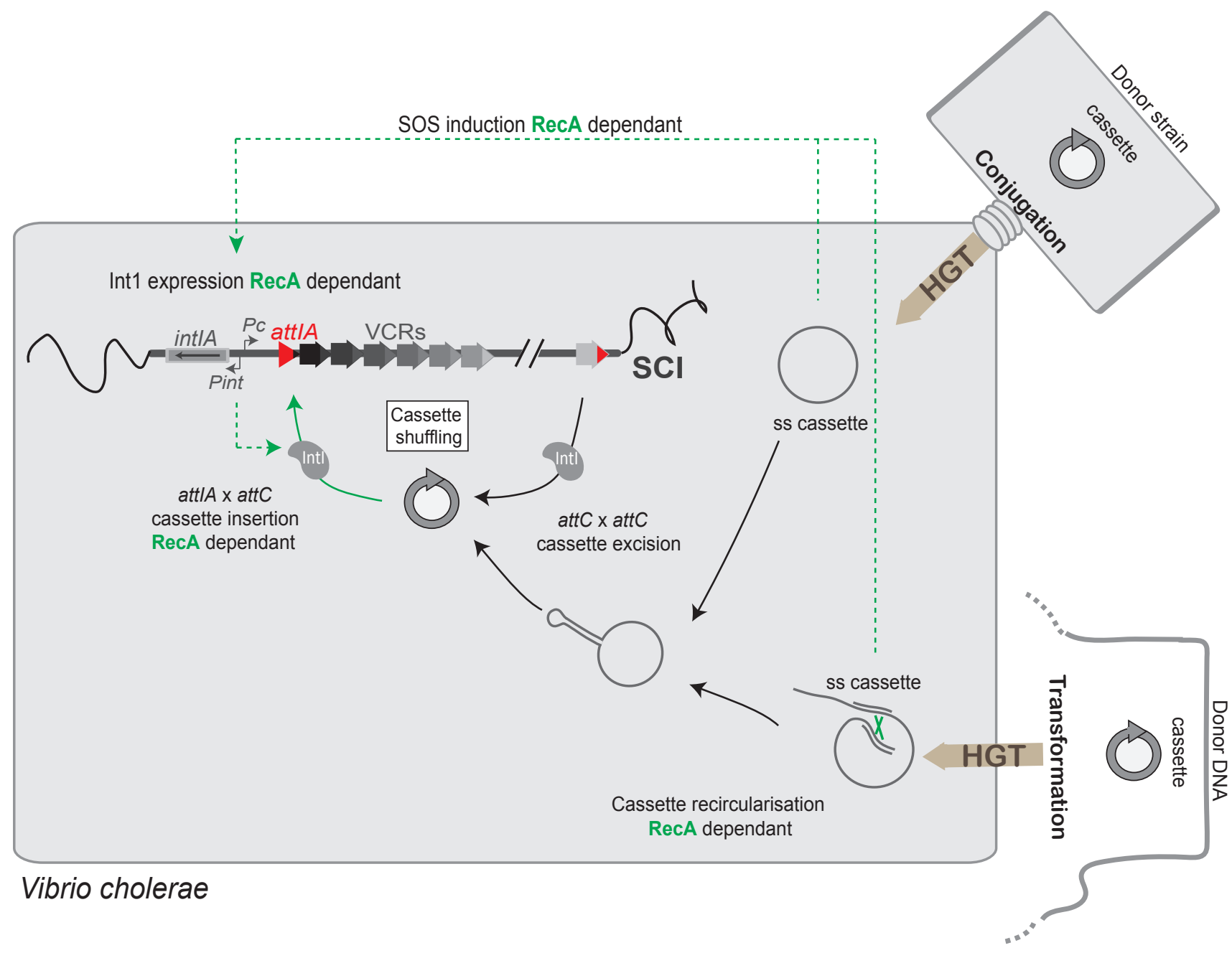

Figure 8 